\title{
On a Family of Multivariate Modified Humbert Polynomials
}

\author{
Rabia Aktaş ${ }^{1}$ and Esra Erkuş-Duman ${ }^{2}$ \\ ${ }^{1}$ Department of Mathematics, Faculty of Science, Ankara University, Tandoğan, 06100 Ankara, Turkey \\ ${ }^{2}$ Department of Mathematics, Faculty of Science, Gazi University, Teknikokullar, 06500 Ankara, Turkey
}

Correspondence should be addressed to Rabia Aktaş; raktas@science.ankara.edu.tr

Received 29 April 2013; Accepted 6 June 2013

Academic Editors: F. J. Garcia-Pacheco and V. Privman

Copyright (C) 2013 R. Aktaş and E. Erkuş-Duman. This is an open access article distributed under the Creative Commons Attribution License, which permits unrestricted use, distribution, and reproduction in any medium, provided the original work is properly cited.

\begin{abstract}
This paper attempts to present a multivariable extension of generalized Humbert polynomials. The results obtained here include various families of multilinear and multilateral generating functions, miscellaneous properties, and also some special cases for these multivariable polynomials.
\end{abstract}

\section{Introduction}

The generalized Humbert polynomials are generated by [1]

$$
\left(C-m x t+y t^{m}\right)^{p}=\sum_{n=0}^{\infty} P_{n}(m, x, y, p, C) t^{n}
$$

where $m$ is a positive integer and other parameters are unrestricted in general (see also [2, pages 77,86$]$ and $[3$, $4])$. This definition includes many well-known special polynomials such as Humbert, Louville, Gegenbauer, Legendre, Tchebycheff, Pincherle, and Kinney polynomials.

In this paper, we consider the following multivariable extension of the generalized Humbert polynomials which are completely different from the polynomials introduced in [5]. This class of polynomials is generated by

$$
\begin{aligned}
\left(1-\sum_{i=1}^{r}\left(m_{i} x_{i} t_{i}-y_{i} t_{i}^{m_{i}}\right)\right)^{-\alpha} & \\
= & \sum_{n_{1}, \ldots, n_{r}=0}^{\infty} \Theta_{n_{1}, \ldots, n_{r}}^{(\alpha)}(\mathbf{x}, \mathbf{y}, \mathbf{m}) t_{1}^{n_{1}} \cdots t_{r}^{n_{r}}, \\
& \left(\left|\sum_{i=1}^{r}\left(m_{i} x_{i} \mathrm{t}_{i}-y_{i} t_{i}^{m_{i}}\right)\right|<1\right),
\end{aligned}
$$

where $\mathbf{x}=\left(x_{1}, \ldots, x_{r}\right), \mathbf{y}=\left(y_{1}, \ldots, y_{r}\right), \mathbf{m}=\left(m_{1}, \ldots, m_{r}\right)$ and $m_{i}(i=1,2, \ldots, r)$ is a positive integer. It follows from (2) that

$$
\begin{aligned}
& \Theta_{n_{1}, \ldots, n_{r}}^{(\alpha)}(\mathbf{x}, \mathbf{y}, \mathbf{m}) \\
& =\sum_{k_{1}=0}^{\left[n_{1} / m_{1}\right]} \cdots \sum_{k_{r}=0}^{\left[n_{r} / m_{r}\right]} \frac{(\alpha)_{n_{1}+\cdots+n_{r}-\left(m_{1}-1\right) k_{1}-\cdots-\left(m_{r}-1\right) k_{r}}}{k_{1} ! \cdots k_{r} !\left(n_{1}-m_{1} k_{1}\right) ! \cdots\left(n_{r}-m_{r} k_{r}\right) !} \\
& \quad \times(-1)^{k_{1}+\cdots+k_{r}}\left(m_{1} x_{1}\right)^{n_{1}-m_{1} k_{1}} \cdots\left(m_{r} x_{r}\right)^{n_{r}-m_{r} k_{r}} y_{1}^{k_{1}} \cdots y_{r}^{k_{r}},
\end{aligned}
$$

where $(\lambda)_{k}:=\lambda(\lambda+1) \cdots(\lambda+k-1),(\lambda)_{0}:=1$ is the Pochhammer symbol.

The aim of this paper is to derive various families of multilinear and multilateral generating functions and to give several recurrence relations and expansions in the series of orthogonal polynomials for the family of multivariable polynomials given explicitly by (3). We present some special cases of our results and also obtain some other properties for these special cases.

\section{Bilinear and Bilateral Generating Functions}

In this section, with the help of the similar method as considered in [5-9], we derive several families of bilinear and 
bilateral generating functions for the family of multivariable polynomials generated by (2) and given explicitly by (3).

We begin by stating the following theorem.

Theorem 1. Corresponding to an identically nonvanishing function $\Omega_{\mu}(\mathbf{z})$ of $s$ complex variables $z_{1}, \ldots, z_{s}(s \in \mathbb{N})$ and of complex order $\mu$, let

$$
\Lambda_{\mu, \nu}(\mathbf{z} ; w):=\sum_{k=0}^{\infty} a_{k} \Omega_{\mu+\nu k}(\mathbf{z}) w^{k},
$$

where $\left(a_{k} \neq 0, \quad \mu, v \in \mathbb{C}\right) ; \mathbf{z}=\left(z_{1}, \ldots, z_{s}\right)$. Then, for $p \in \mathbb{N} ; \mathbf{x}=$ $\left(x_{1}, \ldots, x_{r}\right) ; \mathbf{y}=\left(y_{1}, \ldots, y_{r}\right) ; \mathbf{m}=\left(m_{1}, \ldots, m_{r}\right), m_{i} \in \mathbb{N}(i=$ $1,2, \ldots, r)$, one has

$$
\begin{aligned}
\sum_{n_{1}, \ldots, n_{r}=0}^{\infty} \sum_{k=0}^{\left[n_{1} / p\right]} a_{k} \Theta_{n_{1}-p k, n_{2}, \ldots, n_{r}}^{(\alpha)}(\mathbf{x}, \mathbf{y}, \mathbf{m}) \\
\quad \times t_{2}^{n_{2}} \cdots t_{r}^{n_{r}} \Omega_{\mu+\nu k}(\mathbf{z}) \eta^{k} t_{1}^{n_{1}-p k} \\
=\left(1-\sum_{i=1}^{r}\left(m_{i} x_{i} t_{i}-y_{i} t_{i}^{m_{i}}\right)\right)^{-\alpha} \Lambda_{\mu, \nu}(\mathbf{z} ; \eta)
\end{aligned}
$$

provided that each member of (5) exists.

Proof. For convenience, let $S$ denote the first member of the assertion (5) of Theorem 1. Replacing $n_{1}$ by $n_{1}+p k$, we may write that

$$
\begin{aligned}
S & =\sum_{n_{1}, \ldots, n_{r}=0}^{\infty} \sum_{k=0}^{\infty} a_{k} \Theta_{n_{1}, n_{2}, \ldots, n_{r}}^{(\alpha)}(\mathbf{x}, \mathbf{y}, \mathbf{m}) \Omega_{\mu+v k}(\mathbf{z}) \eta^{k} t_{1}^{n_{1}} \cdots t_{r}^{n_{r}} \\
& =\sum_{n_{1}, \ldots, n_{r}=0}^{\infty} \Theta_{n_{1}, n_{2}, \ldots, n_{r}}^{(\alpha)}(\mathbf{x}, \mathbf{y}, \mathbf{m}) t_{1}^{n_{1}} \cdots t_{r}^{n_{r}} \sum_{k=0}^{\infty} a_{k} \Omega_{\mu+\nu k}(\mathbf{z}) \eta^{k} \\
& =\left(1-\sum_{i=1}^{r}\left(m_{i} x_{i} t_{i}-y_{i} t_{i}^{m_{i}}\right)\right)^{-\alpha} \Lambda_{\mu, \nu}(\mathbf{z} ; \eta),
\end{aligned}
$$

which completes the proof.

By using a similar idea, we also get the next result immediately.

Theorem 2. For a nonvanishing function $\varphi_{\lambda_{1}, \ldots, \lambda_{r}}(\mathbf{z})$ of $s$ complex variables $z_{1}, \ldots, z_{s}(s \in \mathbb{N})$ and for $p_{i} \in \mathbb{N}, \lambda_{i}, \eta_{i} \in \mathbb{C}$, $\mathbf{z}=\left(z_{1}, \ldots, z_{s}\right), \mathbf{w}=\left(w_{1}, \ldots, w_{r}\right), \lambda:=\left(\lambda_{1}, \ldots, \lambda_{r}\right), \eta:=$ $\left(\eta_{1}, \ldots, \eta_{r}\right)$ let

$$
\begin{aligned}
& \Omega_{\lambda, \eta, \alpha, \beta, \mathbf{m}}^{\mathbf{n}, \mathbf{p}}(\mathbf{x}, \mathbf{y} ; \mathbf{z} ; \mathbf{w}) \\
& :=\sum_{k_{1}=0}^{\left[n_{1} / p_{1}\right]} \cdots \sum_{k_{r}=0}^{\left[n_{r} / p_{r}\right]} a_{k_{1}, \ldots, k_{r}} \Theta_{n_{1}-p_{1} k_{1}, \ldots, n_{r}-p_{r} k_{r}}^{(\alpha+\beta)}(\mathbf{x}, \mathbf{y}, \mathbf{m}) \\
& \quad \times \varphi_{\lambda_{1}+\eta_{1} k_{1}, \ldots, \lambda_{r}+\eta_{r} k_{r}}(\mathbf{z}) w_{1}^{k_{1}} \cdots w_{r}^{k_{r}},
\end{aligned}
$$

where $a_{k_{1}, \ldots, k_{r}} \neq 0 ; n_{i},(i=1,2, \ldots, r) \in \mathbb{N}_{0} ; \mathbb{N}_{0}:=\mathbb{N} \cup\{0\}$ Then, one has

$$
\begin{gathered}
\sum_{l_{1}=0}^{n_{1}} \ldots \sum_{l_{r}=0}^{n_{r}} \sum_{k_{1}=0}^{\left[l_{1} / p_{1}\right]} \cdots \sum_{k_{r}=0}^{\left[l_{r} / p_{r}\right]} a_{k_{1}, \ldots, k_{r}} \Theta_{n_{1}-l_{1}, \ldots, n_{r}-l_{r}}^{(\alpha)}(\mathbf{x}, \mathbf{y}, \mathbf{m}) \\
\times \Theta_{l_{1}-p_{1} k_{1}, \ldots, l_{r}-p_{r} k_{r}}^{(\beta)}(\mathbf{x}, \mathbf{y}, \mathbf{m}) \\
\times \varphi_{\lambda_{1}+\eta_{1} k_{1}, \ldots, \lambda_{r}+\eta_{r} k_{r}}(\mathbf{z}) \\
\times w_{1}^{k_{1}} \cdots w_{r}^{k_{r}}
\end{gathered}
$$

provided that each member of (8) exists.

\section{Special Cases}

As an application of the above theorems, when the multivariable function $\Omega_{\mu+v k}(\mathbf{z}), \mathbf{z}=\left(z_{1}, \ldots, z_{s}\right), k \in \mathbb{N}_{0}, s \in \mathbb{N}$, is expressed in terms of simpler functions of one and more variables, then we can give further applications of the above theorems. We first set

$$
s=r, \quad \Omega_{\mu+\nu k}(\mathbf{z})=\mathrm{g}_{\mu+\nu k}^{\left(\beta_{1}, \ldots, \beta_{r}\right)}(\mathbf{z})
$$

in Theorem 1, where the Chan-Chyan-Srivastava polynomials $\mathrm{g}_{\mu+\nu k}^{\left(\beta_{1}, \ldots, \beta_{r}\right)}(\mathbf{z})[10]$ are generated by

$$
\begin{aligned}
& \prod_{i=1}^{r}\left(1-x_{i} t\right)^{-\alpha_{i}}=\sum_{n=0}^{\infty} \mathrm{g}_{n}^{\left(\alpha_{1}, \ldots, \alpha_{r}\right)}\left(x_{1}, \ldots, x_{r}\right) t^{n} \\
& \left(\alpha_{i} \in \mathbb{C}(i=1,2, \ldots, r) ;|t|<\min \left\{\left|x_{1}\right|^{-1}, \ldots,\left|x_{r}\right|^{-1}\right\}\right) .
\end{aligned}
$$

We are thus led to the following result which provides a class of bilateral generating functions for the Chan-ChyanSrivastava polynomials and the family of multivariable polynomials given explicitly by (3).

Corollary 3. If $\Lambda_{\mu, \nu}(\mathbf{z} ; w):=\sum_{k=0}^{\infty} a_{k} \mathrm{~g}_{\mu+\nu k}^{\left(\beta_{1}, \ldots, \beta_{r}\right)}(\mathbf{z}) w^{k}, a_{k} \neq 0$, $\mu, \nu \in \mathbb{C}, \mathbf{z}=\left(z_{1}, \ldots, z_{r}\right)$ then, one has

$$
\begin{aligned}
\sum_{n_{1}, \ldots, n_{r}=0}^{\infty} \sum_{k=0}^{\left[n_{1} / p\right]} a_{k} \Theta_{n_{1}-p k, n_{2}, \ldots, n_{r}}^{(\alpha)}(\mathbf{x}, \mathbf{y}, \mathbf{m}) \\
\quad \times t_{2}^{n_{2}} \cdots t_{r}^{n_{r}} \mathrm{~g}_{\mu+\nu k}^{\left(\beta_{1}, \ldots, \beta_{r}\right)}(\mathbf{z}) \eta^{k} t_{1}^{n_{1}-p k} \\
=\left(1-\sum_{i=1}^{r}\left(m_{i} x_{i} t_{i}-y_{i} t_{i}^{m_{i}}\right)\right)^{-\alpha} \Lambda_{\mu, \nu}(\mathbf{z} ; \eta)
\end{aligned}
$$

provided that each member of (11) exists. 
Remark 4. Using the generating relation (10) for the ChanChyan-Srivastava polynomials and getting $a_{k}=1, \mu=0, v=$ 1 , we find that

$$
\begin{gathered}
\sum_{n_{1}, \ldots, n_{r}=0}^{\infty} \sum_{k=0}^{\left[n_{1} / p\right]} \Theta_{n_{1}-p k, n_{2}, \ldots, n_{r}}^{(\alpha)}(\mathbf{x}, \mathbf{y}, \mathbf{m}) t_{2}^{n_{2}} \cdots t_{r}^{n_{r}} \mathrm{~g}_{k}^{\left(\beta_{1}, \ldots, \beta_{r}\right)}(\mathbf{z}) \\
\times \eta^{k} t_{1}^{n_{1}-p k} \\
=\left(1-\sum_{i=1}^{r}\left(m_{i} x_{i} t_{i}-y_{i} t_{i}^{m_{i}}\right)\right)^{-\alpha} \prod_{i=1}^{r}\left(1-z_{i} \eta\right)^{-\beta_{i}} \\
|\eta|<\min \left\{\left|z_{1}\right|^{-1}, \ldots,\left|z_{r}\right|^{-1}\right\},\left|\sum_{i=1}^{r}\left(m_{i} x_{i} t_{i}-y_{i} t_{i}^{m_{i}}\right)\right|<1
\end{gathered}
$$

On the other hand, choosing $s=2 r$ and

$$
\varphi_{\lambda_{1}+\eta_{1} k_{1}, \ldots, \lambda_{r}+\eta_{r} k_{r}}(\mathbf{z})=\Theta_{\lambda_{1}+\eta_{1} k_{1}, \ldots, \lambda_{r}+\eta_{r} k_{r}}^{(\gamma)}(\mathbf{u}, \mathbf{v}, \mathbf{m})
$$

$\lambda_{i}, \eta_{i}(i=1,2, \ldots, r) \in \mathbb{N}_{0}, \mathbf{u}=\left(u_{1}, \ldots, u_{r}\right), \mathbf{v}=\left(v_{1}\right.$, $\left.\ldots, v_{r}\right)$ in Theorem 2, we obtain the following class of bilinear generating functions for the family of multivariable polynomials given explicitly by (3).

Corollary 5. If

$$
\begin{aligned}
& \Lambda_{\lambda, \eta, \alpha, \beta, \gamma, \mathbf{m}}^{\mathbf{n , p}}(\mathbf{x}, \mathbf{y} ; \mathbf{u}, \mathbf{v} ; \mathbf{w}) \\
& \quad=\sum_{k_{1}=0}^{\left[n_{1} / p_{1}\right]} \cdots \sum_{k_{r}=0}^{\left[n_{r} / p_{r}\right]} a_{k_{1}, \ldots, k_{r}} \Theta_{n_{1}-p_{1} k_{1}, \ldots, n_{r}-p_{r} k_{r}}^{(\alpha+\beta)}(\mathbf{x}, \mathbf{y}, \mathbf{m}) \\
& \quad \times \Theta_{\lambda_{1}+\eta_{1} k_{1}, \ldots, \lambda_{r}+\eta_{r} k_{r}}^{(\gamma)}(\mathbf{u}, \mathbf{v}, \mathbf{m}) w_{1}^{k_{1}} \cdots w_{r}^{k_{r}},
\end{aligned}
$$

where $a_{k_{1}, \ldots, k_{r}} \neq 0 ; p_{i} \in \mathbb{N} ; n_{i}, \lambda_{i}, \eta_{i} \in \mathbb{N}_{0}, i=1,2, \ldots, r$, then, we get

$$
\begin{aligned}
\sum_{l_{1}=0}^{n_{1}} \ldots \sum_{l_{r}=0}^{n_{r}} \sum_{k_{1}=0}^{\left[l_{1} / p_{1}\right]} \ldots \sum_{k_{r}=0}^{\left[l_{r} / p_{r}\right]} a_{k_{1}, \ldots, k_{r}} \Theta_{n_{1}-l_{1}, \ldots, n_{r}-l_{r}}^{(\alpha)}(\mathbf{x}, \mathbf{y}, \mathbf{m}) \\
\times \Theta_{l_{1}-p_{1} k_{1}, \ldots, l_{r}-p_{r} k_{r}}^{(\beta)}(\mathbf{x}, \mathbf{y}, \mathbf{m}) \\
\times \Theta_{\lambda_{1}+\eta_{1} k_{1}, \ldots, \lambda_{r}+\eta_{r} k_{r}}^{(\gamma)}(\mathbf{u}, \mathbf{v}, \mathbf{m}) \\
\times w_{1}^{k_{1}} \cdots w_{r}^{k_{r}} \\
=\Lambda_{\lambda, \eta, \alpha, \beta, \gamma, \mathbf{m}}^{\mathbf{n}, \mathbf{p}}(\mathbf{x}, \mathbf{y} ; \mathbf{u}, \mathbf{v} ; \mathbf{w})
\end{aligned}
$$

provided that each member of (15) exists.

Furthermore, for every suitable choice of the coefficients $a_{k}\left(k \in \mathbb{N}_{0}\right)$, if the multivariable functions $\Omega_{\mu+\nu k}(\mathbf{y})$ and $\varphi_{\lambda_{1}+\eta_{1} k_{1}, \ldots, \lambda_{r}+\eta_{r} k_{r}}(\mathbf{y}), \mathbf{y}=\left(y_{1}, \ldots, y_{s}\right),(s \in \mathbb{N})$, are expressed as an appropriate product of several simpler functions, the assertions of Theorems 1 and 2 can be applied in order to derive various families of multilinear and multilateral generating functions for the family of multivariable polynomials given explicitly by (3).

\section{Some Miscellaneous Properties}

In this section, we now discuss some further properties of the family of multivariable polynomials given by (3). We start with the following theorems.

Theorem 6. Let $\Psi_{n_{1}, \ldots, n_{r}}(\mathbf{x}, \mathbf{y}, \mathbf{m})$ be a family of functions generated by

$$
\begin{array}{r}
G\left(m_{1} x_{1} t_{1}-y_{1} t_{1}^{m_{1}}, \ldots, m_{r} x_{r} t_{r}-y_{r} t_{r}^{m_{r}}\right) \\
=\sum_{n_{1}, \ldots, n_{r}=0}^{\infty} \Psi_{n_{1}, \ldots, n_{r}}(\mathbf{x}, \mathbf{y}, \mathbf{m}) t_{1}^{n_{1}} \cdots t_{r}^{n_{r}} .
\end{array}
$$

The following relations hold:

$$
\begin{aligned}
n_{i} \Psi_{n_{1}, \ldots, n_{r}}(\mathbf{x}, \mathbf{y}, \mathbf{m})= & x_{i} \frac{\partial}{\partial x_{i}} \Psi_{n_{1}, \ldots, n_{r}}(\mathbf{x}, \mathbf{y}, \mathbf{m}) \\
& -y_{i} \frac{\partial}{\partial x_{i}} \Psi_{n_{1}, \ldots, n_{i-1}, n_{i}-m_{i}+1, n_{i+1}, \ldots, n_{r}}(\mathbf{x}, \mathbf{y}, \mathbf{m})
\end{aligned}
$$

for $n_{i} \geq m_{i}-1, i=1,2, \ldots, r ; n_{j} \geq 0, j \neq i$ and

$$
n_{i} \Psi_{n_{1}, \ldots, n_{r}}(\mathbf{x}, \mathbf{y}, \mathbf{m})=x_{i} \frac{\partial}{\partial x_{i}} \Psi_{n_{1}, \ldots, n_{r}}(\mathbf{x}, \mathbf{y}, \mathbf{m})
$$

for $n_{i}=0,1, \ldots, m_{i}-2, i=1,2, \ldots, r ; n_{j} \geq 0, j \neq i$. Also, one finds that

$$
\begin{aligned}
m_{i} x_{i} \frac{\partial}{\partial y_{i}} & \Psi_{n_{1}, \ldots, n_{r}}(\mathbf{x}, \mathbf{y}, \mathbf{m})-m_{i} y_{i} \frac{\partial}{\partial y_{i}} \\
& \times \Psi_{n_{1}, \ldots, n_{i-1}, n_{i}-m_{i}+1, n_{i+1}, \ldots, n_{r}}(\mathbf{x}, \mathbf{y}, \mathbf{m}) \\
= & -\left(n_{i}-m_{i}+1\right) \Psi_{n_{1}, \ldots, n_{i-1}, n_{i}-m_{i}+1, n_{i+1}, \ldots, n_{r}}(\mathbf{x}, \mathbf{y}, \mathbf{m})
\end{aligned}
$$

for $n_{i} \geq m_{i}-1, i=1,2, \ldots, r ; n_{j} \geq 0, j \neq i$ and

$$
\frac{\partial}{\partial y_{i}} \Psi_{n_{1}, \ldots, n_{r}}(\mathbf{x}, \mathbf{y}, \mathbf{m})=0
$$

for $n_{i}=0,1, \ldots, m_{i}-2, i=1,2, \ldots, r ; n_{j} \geq 0, j \neq i$. 
Proof. Fix $i=1,2, \ldots, r$. Then, by differentiating (16) with respect to $x_{i}$ and $t_{i}$, after making necessary calculations we obtain that

$$
\begin{gathered}
\sum_{n_{1}, \ldots, n_{r}=0}^{\infty} n_{i} \Psi_{n_{1}, \ldots, n_{r}}(\mathbf{x}, \mathbf{y}, \mathbf{m}) t_{1}^{n_{1}} \cdots t_{r}^{n_{r}} \\
=\left(x_{i}-y_{i} t_{i}^{m_{i}-1}\right) \sum_{n_{1}, \ldots, n_{r}=0}^{\infty} \frac{\partial}{\partial x_{i}} \Psi_{n_{1}, \ldots, n_{r}}(\mathbf{x}, \mathbf{y}, \mathbf{m}) t_{1}^{n_{1}} \cdots t_{r}^{n_{r}} \\
=x_{i} \sum_{n_{1}, \ldots, n_{r}=0}^{\infty} \frac{\partial}{\partial x_{i}} \Psi_{n_{1}, \ldots, n_{r}}(\mathbf{x}, \mathbf{y}, \mathbf{m}) t_{1}^{n_{1}} \cdots t_{r}^{n_{r}} \\
\quad-y_{i} \sum_{n_{1}, \ldots, n_{i-1}, n_{i+1}, \ldots, n_{r}=0}^{\infty} \frac{\partial}{\partial x_{i}} \Psi_{n_{1}, \ldots, n_{i-1}, n_{i}-m_{i}+1, n_{i+1}, \ldots, n_{r}}(\mathbf{x}, \mathbf{y}, \mathbf{m}) \\
\times t_{1}^{n_{1}} \cdots t_{r}^{n_{r}} .
\end{gathered}
$$

Comparing the coefficients of $t_{1}^{n_{1}} \cdots t_{r}^{n_{r}}$, we obtain (17) and (18) for the fixed $i$. Similarly, if we differentiate (16) with respect to $y_{i}$ and $t_{i}$, we can find the relation (19).

Theorem 7. If $\Psi_{n_{1}, \ldots, n_{r}}(\mathbf{x}, \mathbf{y}, \mathbf{m})$ is a family of functions generated by (16), then it satisfies the relations

$$
\begin{aligned}
-m_{i} & \frac{\partial}{\partial y_{i}} \Psi_{n_{1}, \ldots, n_{i-1}, n_{i}-1, n_{i+1}, \ldots, n_{r}}(\mathbf{x}, \mathbf{y}, \mathbf{m}) \\
& =\frac{\partial}{\partial x_{i}} \Psi_{n_{1}, \ldots, n_{i-1}, n_{i}-m_{i}, n_{i+1}, \ldots, n_{r}}(\mathbf{x}, \mathbf{y}, \mathbf{m})
\end{aligned}
$$

for $n_{i} \geq m_{i}, i=1,2, \ldots, r ; n_{j} \geq 0, j \neq i$ and

$$
\frac{\partial}{\partial y_{i}} \Psi_{n_{1}, \ldots, n_{i-1}, n_{i}-1, n_{i+1}, \ldots, n_{r}}(\mathbf{x}, \mathbf{y}, \mathbf{m})=0
$$

for $n_{i}=1,2, \ldots, m_{i}-1, i=1,2, \ldots, r ; n_{j} \geq 0, j \neq i$.

Proof. By comparing the derivatives of (16) with respect to $x_{i}$ and $y_{i}$, we have

$$
\begin{aligned}
-m_{i} t_{i} & \sum_{n_{1}, \ldots, n_{r}=0}^{\infty} \frac{\partial}{\partial y_{i}} \Psi_{n_{1}, \ldots, n_{r}}(\mathbf{x}, \mathbf{y}, \mathbf{m}) t_{1}^{n_{1}} \cdots t_{r}^{n_{r}} \\
= & t_{i}^{m_{i}} \sum_{n_{1}, \ldots, n_{r}=0}^{\infty} \frac{\partial}{\partial x_{i}} \Psi_{n_{1}, \ldots, n_{r}}(\mathbf{x}, \mathbf{y}, \mathbf{m}) t_{1}^{n_{1}} \cdots t_{r}^{n_{r}},
\end{aligned}
$$

which implies that

$$
\begin{aligned}
-m_{i} & \frac{\partial}{\partial y_{i}} \Psi_{n_{1}, \ldots, n_{i-1}, n_{i}-1, n_{i+1}, \ldots, n_{r}}(\mathbf{x}, \mathbf{y}, \mathbf{m}) \\
& =\frac{\partial}{\partial x_{i}} \Psi_{n_{1}, \ldots, n_{i-1}, n_{i}-m_{i}, n_{i+1}, \ldots, n_{r}}(\mathbf{x}, \mathbf{y}, \mathbf{m})
\end{aligned}
$$

for $n_{i} \geq m_{i}, i=1,2, \ldots, r ; n_{j} \geq 0, j \neq i$ and

$$
\frac{\partial}{\partial y_{i}} \Psi_{n_{1}, \ldots, n_{i-1}, n_{i}-1, n_{i+1}, \ldots, n_{r}}(\mathbf{x}, \mathbf{y}, \mathbf{m})=0
$$

for $n_{i}=1,2, \ldots, m_{i}-1, i=1,2, \ldots, r ; n_{j} \geq 0, j \neq i$. Thus, the proof is completed.

As a result of these theorems, if we choose

$$
\begin{gathered}
G\left(m_{1} x_{1} t_{1}-y_{1} t_{1}^{m_{1}}, \ldots, m_{r} x_{r} t_{r}-y_{r} t_{r}^{m_{r}}\right) \\
=\left(1-\sum_{i=1}^{r}\left(m_{i} x_{i} t_{i}-y_{i} t_{i}^{m_{i}}\right)\right)^{-\alpha},
\end{gathered}
$$

then we can give some recurrence relations for the family of multivariable polynomials given explicitly by (3). In the view of Theorem 6, we get

Corollary 8. For the family of multivariable polynomials generated by (2), the following relations hold

$$
\begin{aligned}
n_{i} \Theta_{n_{1}, \ldots, n_{r}}^{(\alpha)}(\mathbf{x}, \mathbf{y}, \mathbf{m})= & x_{i} \frac{\partial}{\partial x_{i}} \Theta_{n_{1}, \ldots, n_{r}}^{(\alpha)}(\mathbf{x}, \mathbf{y}, \mathbf{m}) \\
& -y_{i} \frac{\partial}{\partial x_{i}} \Theta_{n_{1}, \ldots, n_{i-1}, n_{i}-m_{i}+1, n_{i+1}, \ldots, n_{r}}^{(\alpha)}(\mathbf{x}, \mathbf{y}, \mathbf{m}) \\
m_{i} x_{i} \frac{\partial}{\partial y_{i}} \Theta_{n_{1}, \ldots, n_{r}}^{(\alpha)}(\mathbf{x}, \mathbf{y}, \mathbf{m})-m_{i} y_{i} \frac{\partial}{\partial y_{i}} & \\
& \times \Theta_{n_{1}, \ldots, n_{i-1}, n_{i}-m_{i}+1, n_{i+1}, \ldots, n_{r}}^{(\alpha)}(\mathbf{x}, \mathbf{y}, \mathbf{m}) \\
= & -\left(n_{i}-m_{i}+1\right) \Theta_{n_{1}, \ldots, n_{i-1}, n_{i}-m_{i}+1, n_{i+1}, \ldots, n_{r}}^{(\alpha)}(\mathbf{x}, \mathbf{y}, \mathbf{m})
\end{aligned}
$$

for $n_{i} \geq m_{i}-1, i=1,2, \ldots, r ; n_{j} \geq 0, j \neq i$. Also, for $n_{i}=$ $0,1, \ldots, m_{i}-2, i=1,2, \ldots, r ; n_{j} \geq 0, j \neq i$, we have

$$
\begin{gathered}
n_{i} \Theta_{n_{1}, \ldots, n_{r}}^{(\alpha)}(\mathbf{x}, \mathbf{y}, \mathbf{m})=x_{i} \frac{\partial}{\partial x_{i}} \Theta_{n_{1}, \ldots, n_{r}}^{(\alpha)}(\mathbf{x}, \mathbf{y}, \mathbf{m}), \\
\frac{\partial}{\partial y_{i}} \Theta_{n_{1}, \ldots, n_{r}}^{(\alpha)}(\mathbf{x}, \mathbf{y}, \mathbf{m})=0 .
\end{gathered}
$$

Corollary 9. By summing the relations given by (28) and (29), respectively, for $i=1,2, \ldots, r$, we get

$$
\begin{aligned}
& \sum_{i=1}^{r} n_{i} \Theta_{n_{1}, \ldots, n_{r}}^{(\alpha)}(\mathbf{x}, \mathbf{y}, \mathbf{m}) \\
&=\sum_{i=1}^{r} x_{i} \frac{\partial}{\partial x_{i}} \Theta_{n_{1}, \ldots, n_{r}}^{(\alpha)}(\mathbf{x}, \mathbf{y}, \mathbf{m}) \\
&-\sum_{i=1}^{r} y_{i} \frac{\partial}{\partial x_{i}} \Theta_{n_{1}, \ldots, n_{i-1}, n_{i}-m_{i}+1, n_{i+1}, \ldots, n_{r}}^{(\alpha)}(\mathbf{x}, \mathbf{y}, \mathbf{m}),
\end{aligned}
$$




$$
\begin{aligned}
& \sum_{i=1}^{r} m_{i} x_{i} \frac{\partial}{\partial y_{i}} \Theta_{n_{1}, \ldots, n_{r}}^{(\alpha)}(\mathbf{x}, \mathbf{y}, \mathbf{m}) \\
&-\sum_{i=1}^{r} m_{i} y_{i} \frac{\partial}{\partial y_{i}} \Theta_{n_{1}, \ldots, n_{i-1}, n_{i}-m_{i}+1, n_{i+1}, \ldots, n_{r}}^{(\alpha)}(\mathbf{x}, \mathbf{y}, \mathbf{m}) \\
&=-\sum_{i=1}^{r}\left(n_{i}-m_{i}+1\right) \\
& \quad \times \Theta_{n_{1}, \ldots, n_{i-1}, n_{i}-m_{i}+1, n_{i+1}, \ldots, n_{r}}^{(\alpha)}(\mathbf{x}, \mathbf{y}, \mathbf{m})
\end{aligned}
$$

for $n_{i} \geq m_{i}-1, i=1,2, \ldots, r ; n_{j} \geq 0, j \neq i$.

Similarly, as a consequence of Theorem 7 , we can give the next result at once.

Corollary 10. Other recurrence relations for the family of multivariable polynomials $\Theta_{n_{1}, \ldots, n_{r}}^{(\alpha)}(\mathbf{x}, \mathbf{y}, \mathbf{m})$ are

$$
\begin{aligned}
-m_{i} & \frac{\partial}{\partial y_{i}} \Theta_{n_{1}, \ldots, n_{i-1}, n_{i}-1, n_{i+1}, \ldots, n_{r}}^{(\alpha)}(\mathbf{x}, \mathbf{y}, \mathbf{m}) \\
\quad & \frac{\partial}{\partial x_{i}} \Theta_{n_{1}, \ldots, n_{i-1}, n_{i}-m_{i}, n_{i+1}, \ldots, n_{r}}^{(\alpha)}(\mathbf{x}, \mathbf{y}, \mathbf{m})
\end{aligned}
$$

for $n_{i} \geq m_{i}, i=1,2, \ldots, r ; n_{j} \geq 0, j \neq i$ and

$$
\frac{\partial}{\partial y_{i}} \Theta_{n_{1}, \ldots, n_{i-1}, n_{i}-1, n_{i+1}, \ldots, n_{r}}^{(\alpha)}(\mathbf{x}, \mathbf{y}, \mathbf{m})=0
$$

for $n_{i}=1,2, \ldots, m_{i}-1, i=1,2, \ldots, r ; n_{j} \geq 0, j \neq i$.

Theorem 11. The generating relation (2) yields the following addition formula for the family of multivariable polynomials given by (3)

$$
\begin{array}{r}
\Theta_{n_{1}, \ldots, n_{r}}^{(\alpha+\beta)}(\mathbf{x}, \mathbf{y}, \mathbf{m})=\sum_{k_{1}=0}^{n_{1}} \cdots \sum_{k_{r}=0}^{n_{r}} \Theta_{n_{1}-k_{1}, \ldots, n_{r}-k_{r}}^{(\alpha)}(\mathbf{x}, \mathbf{y}, \mathbf{m}) \\
\times \Theta_{k_{1}, \ldots, k_{r}}^{(\beta)}(\mathbf{x}, \mathbf{y}, \mathbf{m}) .
\end{array}
$$

Proof. From (2), we have

$$
\begin{aligned}
\sum_{n_{1}, \ldots, n_{r}=0}^{\infty} \Theta_{n_{1}, \ldots, n_{r}}^{(\alpha+\beta)}(\mathbf{x}, \mathbf{y}, \mathbf{m}) t_{1}^{n_{1}} \cdots t_{r}^{n_{r}} \\
=\left(1-\sum_{i=1}^{r}\left(m_{i} x_{i} t_{i}-y_{i} t_{i}^{m_{i}}\right)\right)^{-(\alpha+\beta)} \\
=\left(1-\sum_{i=1}^{r}\left(m_{i} x_{i} t_{i}-y_{i} t_{i}^{m_{i}}\right)\right)^{-\alpha} \\
\quad \times\left(1-\sum_{i=1}^{r}\left(m_{i} x_{i} t_{i}-y_{i} t_{i}^{m_{i}}\right)\right)^{-\beta}
\end{aligned}
$$

$$
\begin{aligned}
& =\sum_{n_{1}, \ldots, n_{r}=0}^{\infty} \Theta_{n_{1}, \ldots, n_{r}}^{(\alpha)}(\mathbf{x}, \mathbf{y}, \mathbf{m}) t_{1}^{n_{1}} \cdots t_{r}^{n_{r}} \\
& \quad \times \sum_{k_{1}, \ldots, k_{r}=0}^{\infty} \Theta_{k_{1}, \ldots, k_{r}}^{(\beta)}(\mathbf{x}, \mathbf{y}, \mathbf{m}) t_{1}^{k_{1}} \cdots t_{r}^{k_{r}} .
\end{aligned}
$$

Replacing $n_{i}$ by $n_{i}-k_{i}, i=1,2, \ldots, r$, the right-hand side of the last equality is

$$
\begin{aligned}
\sum_{n_{1}, \ldots, n_{r}=0}^{\infty} \sum_{k_{1}=0}^{n_{1}} \cdots \sum_{k_{r}=0}^{n_{r}} & \Theta_{n_{1}-k_{1}, \ldots, n_{r}-k_{r}}^{(\alpha)}(\mathbf{x}, \mathbf{y}, \mathbf{m}) \\
& \times \Theta_{k_{1}, \ldots, k_{r}}^{(\beta)}(\mathbf{x}, \mathbf{y}, \mathbf{m}) t_{1}^{n_{1}} \cdots t_{r}^{n_{r}} .
\end{aligned}
$$

Comparing the coefficients of $t_{1}^{n_{1}} \cdots t_{r}^{n_{r}}$ completes the proof.

We now give expansions of the family of multivariable polynomials $\Theta_{n_{1}, \ldots, n_{r}}^{(\alpha)}(\mathbf{x}, \mathbf{y}, \mathbf{m})$ given explicitly by (3) in series of Legendre, Gegenbauer, Hermite, and Laguerre polynomials.

Theorem 12. Expansions of $\Theta_{n_{1}, \ldots, n_{r}}^{(\alpha)}(\mathbf{x}, \mathbf{y}, \mathbf{m})$ in series of Legendre, Gegenbauer, Hermite, and Laguerre polynomials are as follows

$$
\begin{aligned}
& \Theta_{n_{1}, \ldots, n_{r}}^{(\alpha)}(\mathbf{x}, \mathbf{y}, \mathbf{m}) \\
& =\prod_{i=1}^{r}\left\{\sum_{k_{i}=0}^{\left[n_{i} / m_{i}\right]} \sum_{s_{i}=0}^{\left[\left(n_{i}-m_{i} k_{i}\right) / 2\right]} \frac{\left(2 n_{i}-2 m_{i} k_{i}-4 s_{i}+1\right)}{k_{i} ! s_{i} !(3 / 2)_{n_{i}-m_{i} k_{i}-s_{i}}}\right. \\
& \left.\times(-1)^{k_{i}} y_{i}^{k_{i}} P_{n_{i}-m_{i} k_{i}-2 s_{i}}\left(\frac{m_{i} x_{i}}{2}\right)\right\} \\
& \times(\alpha)_{n_{1}+\cdots+n_{r}-\left(m_{1}-1\right) k_{1}-\cdots-\left(m_{r}-1\right) k_{r}}, \\
& \Theta_{n_{1}, \ldots, n_{r}}^{(\alpha)}(\mathbf{x}, \mathbf{y}, \mathbf{m}) \\
& =\prod_{i=1}^{r}\left\{\sum_{k_{i}=0}^{\left[n_{i} / m_{i}\right]} \sum_{s_{i}=0}^{\left[\left(n_{i}-m_{i} k_{i}\right) / 2\right]} \frac{\left(\nu_{i}+n_{i}-m_{i} k_{i}-2 s_{i}\right)}{k_{i} ! s_{i} !\left(v_{i}\right)_{n_{i}-m_{i} k_{i}-s_{i}+1}}\right. \\
& \left.\times\left(-y_{i}\right)^{k_{i}} C_{n_{i}-m_{i} k_{i}-2 s_{i}}^{\nu_{i}}\left(\frac{m_{i} x_{i}}{2}\right)\right\} \\
& \times(\alpha)_{n_{1}+\cdots+n_{r}-\left(m_{1}-1\right) k_{1}-\cdots-\left(m_{r}-1\right) k_{r}}, \\
& \Theta_{n_{1}, \ldots, n_{r}}^{(\alpha)}(\mathbf{x}, \mathbf{y}, \mathbf{m}) \\
& =\prod_{i=1}^{r}\left\{\sum_{k_{i}=0}^{\left[n_{i} / m_{i}\right]} \sum_{s_{i}=0}^{\left[\left(n_{i}-m_{i} k_{i}\right) / 2\right]} \frac{\left(-y_{i}\right)^{k_{i}} H_{n_{i}-m_{i} k_{i}-2 s_{i}}\left(m_{i} x_{i} / 2\right)}{k_{i} ! s_{i} !\left(n_{i}-m_{i} k_{i}-2 s_{i}\right) !}\right\} \\
& \times(\alpha)_{n_{1}+\cdots+n_{r}-\left(m_{1}-1\right) k_{1}-\cdots-\left(m_{r}-1\right) k_{r}},
\end{aligned}
$$




$$
\begin{aligned}
& \Theta_{n_{1}, \ldots, n_{r}}^{(\alpha)}(\mathbf{x}, \mathbf{y}, \mathbf{m}) \\
& =\prod_{i=1}^{r}\left\{\sum_{k_{i}=0}^{\left[n_{i} / m_{i}\right]} \sum_{s_{i}=0}^{n_{i}-m_{i} k_{i}} \frac{\left(\beta_{i}+1\right)_{n_{i}-m_{i} k_{i}} 2^{n_{i}-m_{i} k_{i}}(-1)^{s_{i}}}{k_{i} !\left(n_{i}-m_{i} k_{i}-s_{i}\right) !\left(\beta_{i}+1\right)_{s_{i}}}\right. \\
& \left.\times\left(-y_{i}\right)^{k_{i}} L_{s_{i}}^{\left(\beta_{i}\right)}\left(\frac{m_{i} x_{i}}{2}\right)\right\} \\
& \times(\alpha)_{n_{1}+\cdots+n_{r}-\left(m_{1}-1\right) k_{1}-\cdots-\left(m_{r}-1\right) k_{r}}
\end{aligned}
$$

Proof. By (2), we get

$$
\begin{gathered}
\sum_{n_{1}, \ldots, n_{r}=0}^{\infty} \Theta_{n_{1}, \ldots, n_{r}}^{(\alpha)}(\mathbf{x}, \mathbf{y}, \mathbf{m}) t_{1}^{n_{1}} \cdots t_{r}^{n_{r}} \\
=\left(1-\sum_{i=1}^{r}\left(m_{i} x_{i} t_{i}-y_{i} t_{i}^{m_{i}}\right)\right)^{-\alpha} \\
=\sum_{n_{1}, \ldots, n_{r}=0}^{\infty} \sum_{k_{1}, \ldots, k_{r}=0}^{\infty} \frac{(\alpha)_{n_{1}+\cdots+n_{r}+k_{1}+\cdots+k_{r}}}{k_{1} ! \cdots k_{r} ! n_{1} ! \cdots n_{r} !}(-1)^{k_{1}+\cdots+k_{r}} \\
\quad \times\left(m_{1} x_{1}\right)^{n_{1}} \cdots\left(m_{r} x_{r}\right)^{n_{r}} y_{1}^{k_{1}} \cdots y_{r}^{k_{r}} \\
\quad \times t_{1}^{n_{1}+m_{1} k_{1}} \cdots t_{r}^{n_{r}+m_{r} k_{r}} .
\end{gathered}
$$

If we use the result in [11, page 181]

$$
\frac{(m x)^{n}}{n !}=\sum_{s=0}^{[n / 2]} \frac{(2 n-4 s+1) P_{n-2 s}(m x / 2)}{s !(3 / 2)_{n-s}}
$$

we can write that

$$
\begin{aligned}
\sum_{n_{1}, \ldots, n_{r}=0}^{\infty} \Theta_{n_{1}, \ldots, n_{r}}^{(\alpha)}(\mathbf{x}, \mathbf{y}, \mathbf{m}) t_{1}^{n_{1}} \cdots t_{r}^{n_{r}} \\
=\prod_{i=1}^{r}\left\{\sum_{n_{i}=0}^{\infty} \sum_{k_{i}=0}^{\infty} \sum_{s_{i}=0}^{\left[n_{i} / 2\right]} \frac{\left(2 n_{i}-4 s_{i}+1\right)}{s_{i} ! k_{i} !(3 / 2)_{n_{i}-s_{i}}} P_{n_{i}-2 s_{i}}\left(\frac{m_{i} x_{i}}{2}\right)\right. \\
\left.\times\left(-y_{i}\right)^{k_{i}} t_{i}^{n_{i}+m_{i} k_{i}}\right\}(\alpha)_{n_{1}+\cdots+n_{r}+k_{1}+\cdots+k_{r}} .
\end{aligned}
$$

Getting $n_{i}-m_{i} k_{i}$ instead of $n_{i}$ in the last equality, we have

$$
\begin{gathered}
\sum_{n_{1}, \ldots, n_{r}=0}^{\infty} \Theta_{n_{1}, \ldots, n_{r}}^{(\alpha)}(\mathbf{x}, \mathbf{y}, \mathbf{m}) t_{1}^{n_{1}} \cdots t_{r}^{n_{r}} \\
=\sum_{n_{1}, \ldots, n_{r}=0}^{\infty} \prod_{i=1}^{r}\left\{\sum_{k_{i}=0}^{\left[n_{i} / m_{i}\right]\left[\left(n_{i}-m_{i} k_{i}\right) / 2\right]} \sum_{s_{i}=0}^{\frac{\left(2 n_{i}-2 m_{i} k_{i}-4 s_{i}+1\right)}{s_{i} ! k_{i} !(3 / 2)_{n_{i}-m_{i} k_{i}-s_{i}}}}\right. \\
\left.\times P_{n_{i}-m_{i} k_{i}-2 s_{i}}\left(\frac{m_{i} x_{i}}{2}\right)\left(-y_{i}\right)^{k_{i}} t_{i}^{n_{i}}\right\} \\
\sum_{n_{1}, \ldots, n_{r}=0}^{\infty}\left\{\begin{array}{l}
\prod_{i=1}^{r} \sum_{k_{i}=0}^{\left[n_{i} / m_{i}\right]\left[\left(n_{i}-m_{i} k_{i}\right) / 2\right]} \sum_{s_{i}=0} \frac{\left(2 n_{i}-2 m_{i} k_{i}-4 s_{i}+1\right)}{s_{i} ! k_{i} !(3 / 2)_{n_{i}-m_{i} k_{i}-s_{i}}} \\
\left.\times P_{n_{i}-m_{i} k_{i}-2 s_{i}}\left(\frac{m_{i} x_{i}}{2}\right)\left(-y_{i}\right)^{k_{i}}\right\} \\
\times(\alpha)_{n_{1}+\cdots+n_{r}-\left(m_{1}-1\right) k_{1}-\cdots-\left(m_{r}-1\right) k_{r}} t_{1}^{n_{1}} \cdots t_{r}^{n_{r}} .
\end{array}\right.
\end{gathered}
$$

Comparing the coefficients of $t_{1}^{n_{1}} \cdots t_{r}^{n_{r}}$ gives the desired relation.

In a similar manner, in (39), using the following results, respectively, [11, page 283 (36), page 194 (4), page 207 (2)]

$$
\begin{gathered}
\frac{(m x)^{n}}{n !}=\sum_{k=0}^{[n / 2]} \frac{(\nu+n-2 k) C_{n-2 k}^{v}(m x / 2)}{k !(\nu)_{n+1-k}}, \\
\frac{(m x)^{n}}{n !}=\sum_{k=0}^{[n / 2]} \frac{H_{n-2 k}(m x / 2)}{k !(n-2 k) !} \\
\frac{(m x)^{n}}{n !}=2^{n} \sum_{k=0}^{n} \frac{(-1)^{k}(\alpha+1)_{n} L_{k}^{(\alpha)}(m x / 2)}{(n-k) !(\alpha+1)_{k}},
\end{gathered}
$$

one can easily obtain the other expansions of $\Theta_{n_{1}, \ldots, n_{r}}^{(\alpha)}(\mathbf{x}, \mathbf{y}, \mathbf{m})$ in series of Gegenbauer, Hermite, and Laguerre polynomials.

\section{The Special Cases of $\Theta_{n}^{(\alpha)}(x, y, m)$ and Some Properties}

In this section, we discuss some special cases of the family of multivariable polynomials $\Theta_{n}^{(\alpha)}(\mathbf{x}, \mathbf{y}, \mathbf{m})$ and give their several properties.

5.1. The Case of $m_{i}=2, y_{i}=1, i=1,2, \ldots, r$ in (2). This case gives a multivariable analogue of Gegenbauer polynomials

$$
\begin{aligned}
& \left(1-2 x_{1} t_{1}+t_{1}^{2}-\cdots-2 x_{r} t_{r}+t_{r}^{2}\right)^{-\alpha} \\
& \quad=\sum_{n_{1}, \ldots, n_{r}=0}^{\infty} C_{n_{1}, \ldots, n_{r}}^{(\alpha)}\left(x_{1}, \ldots, x_{r}\right) t_{1}^{n_{1}} \cdots t_{r}^{n_{r}},
\end{aligned}
$$


which reduces to two variables Gegenbauer polynomials given by [12] for $r=2$. Equation (44) yields the following formula:

$$
\begin{gathered}
C_{n_{1}, \ldots, n_{r}}^{(\alpha)}\left(x_{1}, \ldots, x_{r}\right) \\
=\sum_{k_{1}=0}^{\left[n_{1} / 2\right]} \cdots \sum_{k_{r}=0}^{\left[n_{r} / 2\right]}\left(\left((\alpha)_{n_{1}+\cdots+n_{r}-k_{1}-\cdots-k_{r}}\right)\right. \\
\times\left(k_{1} ! \cdots k_{r} !\left(n_{1}-2 k_{1}\right) !\right. \\
\left.\left.\cdots\left(n_{r}-2 k_{r}\right) !\right)^{-1}\right) \\
\times(-1)^{k_{1}+\cdots+k_{r}}\left(2 x_{1}\right)^{n_{1}-2 k_{1}} \cdots\left(2 x_{r}\right)^{n_{r}-2 k_{r}} .
\end{gathered}
$$

It follows that $C_{n_{1}, \ldots, n_{r}}^{(\alpha)}\left(x_{1}, \ldots, x_{r}\right)$ is a polynomial of degree $n_{i}$ with respect to the fixed variable $x_{i}(i=1,2, \ldots, r)$. Thus, $C_{n_{1}, \ldots, n_{r}}^{(\alpha)}\left(x_{1}, \ldots, x_{r}\right)$ is a polynomial of total degree $\left(n_{1}+\right.$ $\cdots+n_{r}$ ) with respect to the variables $x_{1}, \ldots, x_{r}$. Equation (45) also yields

$$
\begin{aligned}
C_{n_{1}, \ldots, n_{r}}^{(\alpha)} & \left(x_{1}, \ldots, x_{r}\right) \\
= & \frac{2^{n_{1}+\cdots+n_{r}}(\alpha)_{n_{1}+\cdots+n_{r}}}{n_{1} ! \cdots n_{r} !} x_{1}^{n_{1}} \cdots x_{r}^{n_{r}}+\Pi\left(x_{1}, \ldots, x_{r}\right),
\end{aligned}
$$

where $\Pi\left(x_{1}, \ldots, x_{r}\right)$ is a polynomial of degree $\left(n_{1}+\cdots+n_{r}-\right.$ 2 ) with respect to the variables $x_{1}, \ldots, x_{r}$. In (44), by getting $x_{1} \rightarrow-x_{1}$ and $t_{1} \rightarrow-t_{1}$, we have

$$
C_{n_{1}, \ldots, n_{r}}^{(\alpha)}\left(-x_{1}, x_{2}, \ldots, x_{r}\right)=(-1)^{n_{1}} C_{n_{1}, \ldots, n_{r}}^{(\alpha)}\left(x_{1}, \ldots, x_{r}\right) .
$$

Similarly, for $i=1,2, \ldots, r$, we get

$$
\begin{gathered}
C_{n_{1}, \ldots, n_{r}}^{(\alpha)}\left(x_{1}, \ldots, x_{i-1},-x_{i}, x_{i+1}, \ldots, x_{r}\right) \\
=(-1)^{n_{i}} C_{n_{1}, \ldots, n_{r}}^{(\alpha)}\left(x_{1}, \ldots, x_{r}\right) .
\end{gathered}
$$

Taking $x_{i} \rightarrow-x_{i}$ and $t_{i} \rightarrow-t_{i}, i=1,2, \ldots, r$, in (44), we obtain

$$
C_{n_{1}, \ldots, n_{r}}^{(\alpha)}\left(-x_{1}, \ldots,-x_{r}\right)=(-1)^{n_{1}+\cdots+n_{r}} C_{n_{1}, \ldots, n_{r}}^{(\alpha)}\left(x_{1}, \ldots, x_{r}\right) .
$$

Theorem 13. For the polynomials $C_{n_{1}, \ldots, n_{r}}^{(\alpha)}\left(x_{1}, \ldots, x_{r}\right)$, one has

$$
C_{2 n_{1}, \ldots, 2 n_{r}}^{(\alpha)}(0,0, \ldots, 0)=\frac{(-1)^{n_{1}+\cdots+n_{r}}(\alpha)_{n_{1}+\cdots+n_{r}}}{n_{1} ! \cdots n_{r} !}
$$

and if at least one of $n_{i}, i=1,2, \ldots, r$, is odd; then

$$
C_{n_{1}, \ldots, n_{r}}^{(\alpha)}(0,0, \ldots, 0)=0 .
$$

Proof. If we set all $x_{i}=0, i=1,2, \ldots, r$ in (44), we have

$$
\left(1+t_{1}^{2}+\cdots+t_{r}^{2}\right)^{-\alpha}=\sum_{n_{1}, \ldots, n_{r}=0}^{\infty} C_{n_{1}, \ldots, n_{r}}^{(\alpha)}(0,0, \ldots, 0) t_{1}^{n_{1}} \cdots t_{r}^{n_{r}}
$$

On the other hand, we get

$$
\begin{aligned}
(1 & \left.+t_{1}^{2}+\cdots+t_{r}^{2}\right)^{-\alpha} \\
& =\sum_{n_{1}, \ldots, n_{r}=0}^{\infty} \frac{(-1)^{n_{1}+\cdots+n_{r}}}{n_{1} ! \cdots n_{r} !}(\alpha)_{n_{1}+\cdots+n_{r}} t_{1}^{2 n_{1}} \cdots t_{r}^{2 n_{r}} .
\end{aligned}
$$

By comparing the coefficients of $t_{1}^{n_{1}} \cdots t_{r}^{n_{r}}$, we obtain the desired.

From the theorems and corollaries given in Section 4, we can give some other properties of $C_{n_{1}, \ldots, n_{r}}^{(\alpha)}\left(x_{1}, \ldots, x_{r}\right)$.

Remark 14. By Corollaries 8 and 9, for the family of multivariable polynomials generated by (44), the following relations:

$$
\begin{aligned}
n_{i} C_{n_{1}, \ldots, n_{r}}^{(\alpha)} & \left(x_{1}, \ldots, x_{r}\right) \\
= & x_{i} \frac{\partial}{\partial x_{i}} C_{n_{1}, \ldots, n_{r}}^{(\alpha)}\left(x_{1}, \ldots, x_{r}\right) \\
& -\frac{\partial}{\partial x_{i}} C_{n_{1}, \ldots, n_{i-1}, n_{i}-1, n_{i+1}, \ldots, n_{r}}^{(\alpha)}\left(x_{1}, \ldots, x_{r}\right), \\
\sum_{i=1}^{r} n_{i} C_{n_{1}, \ldots, n_{r}}^{(\alpha)}\left(x_{1}, \ldots, x_{r}\right) & \\
= & \sum_{i=1}^{r} x_{i} \frac{\partial}{\partial x_{i}} C_{n_{1}, \ldots, n_{r}}^{(\alpha)}\left(x_{1}, \ldots, x_{r}\right) \\
& -\sum_{i=1}^{r} \frac{\partial}{\partial x_{i}} C_{n_{1}, \ldots, n_{i-1}, n_{i}-1, n_{i+1}, \ldots, n_{r}}^{(\alpha)}\left(x_{1}, \ldots, x_{r}\right)
\end{aligned}
$$

hold for $n_{i} \geq 1, i=1,2, \ldots, r$.

Remark 15. From Theorem 11, the multivariable polynomials $C_{n_{1}, \ldots, n_{r}}^{(\alpha)}\left(x_{1}, \ldots, x_{r}\right)$ satisfy the following addition formula:

$$
\begin{array}{r}
C_{n_{1}, \ldots, n_{r}}^{(\alpha+\beta)}\left(x_{1}, \ldots, x_{r}\right)=\sum_{k_{1}=0}^{n_{1}} \ldots \sum_{k_{r}=0}^{n_{r}} C_{n_{1}-k_{1}, \ldots, n_{r}-k_{r}}^{(\alpha)}\left(x_{1}, \ldots, x_{r}\right) \\
\times C_{k_{1}, \ldots, k_{r}}^{(\beta)}\left(x_{1}, \ldots, x_{r}\right) .
\end{array}
$$


Remark 16. As a result of Theorem 12, expansions of $C_{n_{1}, \ldots, n_{r}}^{(\alpha)}$ $\left(x_{1}, \ldots, x_{r}\right)$ in series of Legendre, Gegenbauer, Hermite, and Laguerre polynomials are as follows:

$$
\begin{aligned}
& C_{n_{1}, \ldots, n_{r}}^{(\alpha)}\left(x_{1}, \ldots, x_{r}\right) \\
& =\prod_{i=1}^{r}\left\{\sum_{k_{i}=0}^{\left[n_{i} / 2\right]\left[\left(n_{i}-2 k_{i}\right) / 2\right]} \sum_{s_{i}=0}^{\frac{\left(2 n_{i}-4 k_{i}-4 s_{i}+1\right)}{k_{i} ! s_{i} !(3 / 2)_{n_{i}-2 k_{i}-s_{i}}}}\right. \\
& \left.\times(-1)^{k_{i}} P_{n_{i}-2 k_{i}-2 s_{i}}\left(x_{i}\right)\right\} \\
& \times(\alpha)_{n_{1}+\cdots+n_{r}-k_{1}-\cdots-k_{r}}, \\
& C_{n_{1}, \ldots, n_{r}}^{(\alpha)}\left(x_{1}, \ldots, x_{r}\right) \\
& =\prod_{i=1}^{r}\left\{\sum_{k_{i}=0}^{\left[n_{i} / 2\right]\left[\left(n_{i}-2 k_{i}\right) / 2\right]} \sum_{s_{i}=0}^{\frac{\left(v_{i}+n_{i}-2 k_{i}-2 s_{i}\right)}{k_{i} ! s_{i} !\left(v_{i}\right)_{n_{i}-2 k_{i}-s_{i}+1}}}\right. \\
& \left.\times(-1)^{k_{i}} C_{n_{i}-2 k_{i}-2 s_{i}}^{v_{i}}\left(x_{i}\right)\right\} \\
& \times(\alpha)_{n_{1}+\cdots+n_{r}-k_{1}-\cdots-k_{r}}, \\
& C_{n_{1}, \ldots, n_{r}}^{(\alpha)}\left(x_{1}, \ldots, x_{r}\right) \\
& =\prod_{i=1}^{r}\left\{\sum_{k_{i}=0}^{\left[n_{i} / 2\right]\left[\left(n_{i}-2 k_{i}\right) / 2\right]} \sum_{s_{i}=0}^{(-1)^{k_{i}} H_{n_{i}-2 k_{i}-2 s_{i}}\left(x_{i}\right)} \frac{k_{i} ! s_{i} !\left(n_{i}-2 k_{i}-2 s_{i}\right) !}{\}}\right. \\
& \times(\alpha)_{n_{1}+\cdots+n_{r}-k_{1}-\cdots-k_{r}}, \\
& C_{n_{1}, \ldots, n_{r}}^{(\alpha)}\left(x_{1}, \ldots, x_{r}\right) \\
& =\prod_{i=1}^{r}\left\{\sum_{k_{i}=0}^{\left[n_{i} / 2\right]} \sum_{s_{i}=0}^{n_{i}-2 k_{i}} \frac{\left(\beta_{i}+1\right)_{n_{i}-2 k_{i}} 2^{n_{i}-2 k_{i}}(-1)^{s_{i}}}{k_{i} !\left(n_{i}-2 k_{i}-s_{i}\right) !\left(\beta_{i}+1\right)_{s_{i}}}\right. \\
& \left.\times(-1)^{k_{i}} L_{s_{i}}^{\left(\beta_{i}\right)}\left(x_{i}\right)\right\} \\
& \times(\alpha)_{n_{1}+\cdots+n_{r}-k_{1}-\cdots-k_{r}} .
\end{aligned}
$$

We now give a hypergeometric representation for the multivariable polynomials $C_{n_{1}, \ldots, n_{r}}^{(\alpha)}\left(x_{1}, \ldots, x_{r}\right)$ given by (44).

Theorem 17. The multivariable polynomials $C_{n_{1}, \ldots, n_{r}}^{(\alpha)}\left(x_{1}, \ldots\right.$, $x_{r}$ ) have the following hypergeometric representation:

$$
\begin{aligned}
C_{n_{1}, \ldots, n_{r}}^{(\alpha)} & \left(x_{1}, \ldots, x_{r}\right) \\
& =\frac{(\alpha)_{n_{1}+\cdots+n_{r}}}{n_{1} ! \cdots n_{r} !}\left(2 x_{1}\right)^{n_{1}} \cdots\left(2 x_{r}\right)^{n_{r}}
\end{aligned}
$$

$$
\begin{gathered}
\times F_{B}^{(r)}\left[-\frac{n_{1}}{2}, \ldots,-\frac{n_{r}}{2}, \frac{-n_{1}+1}{2}, \ldots, \frac{-n_{r}+1}{2} ;\right. \\
\left.1-\alpha-n_{1}-\cdots-n_{r} ; \frac{1}{x_{1}^{2}}, \ldots, \frac{1}{x_{r}^{2}}\right], \\
\left(\max \left\{\frac{1}{x_{1}^{2}}, \ldots, \frac{1}{x_{r}^{2}}\right\}<1\right),
\end{gathered}
$$

where $F_{B}^{(r)}$ is a Lauricella function of $r$-variable defined by [13] (see also [2])

$$
\begin{array}{r}
F_{B}^{(r)}\left[a_{1}, \ldots, a_{r}, b_{1}, \ldots, b_{r} ; c ; x_{1}, \ldots, x_{r}\right] \\
=\sum_{n_{1}, \ldots, n_{r}=0}^{\infty} \frac{\left(a_{1}\right)_{n_{1}} \cdots\left(a_{r}\right)_{n_{r}}\left(b_{1}\right)_{n_{1}} \cdots\left(b_{r}\right)_{n_{r}}}{(c)_{n_{1}+\cdots+n_{r}}} \frac{x_{1}^{n_{1}}}{n_{1} !} \cdots \frac{x_{r}^{n_{r}}}{n_{r} !}, \\
\left(\max \left\{\left|x_{1}\right|, \ldots,\left|x_{r}\right|\right\}<1\right) .
\end{array}
$$

Proof. We have the following results from [11]:

$$
\begin{gathered}
(\alpha)_{n-k}=\frac{(-1)^{k}(\alpha)_{n}}{(1-\alpha-n)_{k}}, \quad(-n)_{2 k}=2^{2 k}\left(-\frac{n}{2}\right)_{k}\left(\frac{-n+1}{2}\right)_{k}, \\
(n-2 k) !=\frac{n !}{(-n)_{2 k}} .
\end{gathered}
$$

In view of these relations, the equality (45) can be written as follows:

$$
\begin{aligned}
& C_{n_{1}, \ldots, n_{r}}^{(\alpha)}\left(x_{1}, \ldots, x_{r}\right) \\
&=\sum_{k_{1}=0}^{\left[n_{1} / 2\right]} \cdots \sum_{k_{r}=0}^{\left[n_{r} / 2\right]}(\left((\alpha)_{n_{1}+\cdots+n_{r}} 2^{2\left(k_{1}+\cdots+k_{r}\right)}\left(-\frac{n_{1}}{2}\right)_{k_{1}}\right. \\
& \times\left(\frac{-n_{1}+1}{2}\right)_{k_{1}} \cdots\left(-\frac{n_{r}}{2}\right)_{k_{r}} \\
&\left.\times\left(\frac{-n_{r}+1}{2}\right)_{k_{r}}\right) \\
& \times\left(k_{1} ! \cdots k_{r} ! n_{1} ! \cdots n_{r} !\right. \\
&\left.\left.\times\left(1-\alpha-n_{1}-\cdots-n_{r}\right)_{k_{1}+\cdots+k_{r}}\right)^{-1}\right) \\
& \times \times\left(2 x_{1}\right)^{n_{1}-2 k_{1}} \cdots\left(2 x_{r}\right)^{n_{r}-2 k_{r}} \\
&=\frac{(\alpha)_{n_{1}+\cdots+n_{r}}}{n_{1} ! \cdots n_{r} !}\left(2 x_{1}\right)^{n_{1}} \cdots\left(2 x_{r}\right)^{n_{r}}
\end{aligned}
$$




$$
\begin{aligned}
& \times \sum_{k_{1}=0}^{\left[n_{1} / 2\right]} \cdots \sum_{k_{r}=0}^{\left[n_{r} / 2\right]}\left(\left(-\frac{n_{1}}{2}\right)_{k_{1}}\left(\frac{-n_{1}+1}{2}\right)_{k_{1}}\right. \\
& \left.\cdots\left(-\frac{n_{r}}{2}\right)_{k_{r}}\left(\frac{-n_{r}+1}{2}\right)_{k_{r}}\right) \\
& \times\left(\left(1-\alpha-n_{1}-\cdots-n_{r}\right)_{k_{1}+\cdots+k_{r}}\right. \\
& \left.\left.\times k_{1} ! \cdots k_{r} !\right)^{-1}\right) \\
& \times\left(\frac{1}{x_{1}^{2}}\right)^{k_{1}} \cdots\left(\frac{1}{x_{r}^{2}}\right)^{k_{r}} \\
& =F_{B}^{(r)}\left[-\frac{n_{1}}{2}, \ldots,-\frac{n_{r}}{2}, \frac{-n_{1}+1}{2}, \ldots, \frac{-n_{r}+1}{2}\right. \text {; } \\
& \left.1-\alpha-n_{1}-\cdots-n_{r} ; \frac{1}{x_{1}^{2}}, \ldots, \frac{1}{x_{r}^{2}}\right] \\
& \times \frac{(\alpha)_{n_{1}+\cdots+n_{r}}}{n_{1} ! \cdots n_{r} !}\left(2 x_{1}\right)^{n_{1}} \cdots\left(2 x_{r}\right)^{n_{r}} \text {, }
\end{aligned}
$$

where $\max \left\{1 / x_{1}^{2}, \ldots, 1 / x_{r}^{2}\right\}<1$. The proof is completed.

Remark 18. For $r=2$, Theorem 17 reduces to the known result for two variable Gegenbauer polynomials given by [12].

5.2. The Case of $x_{i}=0, y_{i}=-x_{i}, i=1,2, \ldots, r$ in (2). This case yields

$$
\begin{aligned}
& \left(1-x_{1} t_{1}^{m_{1}}-x_{2} t_{2}^{m_{2}}-\cdots-x_{r} t_{r}^{m_{r}}\right)^{-\alpha} \\
& \quad=\sum_{n_{1}, \ldots, n_{r}=0}^{\infty} U_{n_{1}, \ldots, n_{r}}^{(\alpha)}\left(x_{1}, \ldots, x_{r}\right) t_{1}^{n_{1}} \cdots t_{r}^{n_{r}},
\end{aligned}
$$

which is a different unification from that in [8].

Remark 19. From Corollaries 8 and 9, the multivariable polynomials $U_{n_{1}, \ldots, n_{r}}^{(\alpha)}\left(x_{1}, \ldots, x_{r}\right)$ satisfy

$$
\begin{aligned}
m_{i} x_{i} & \frac{\partial}{\partial x_{i}} U_{n_{1}, \ldots, n_{i-1}, n_{i}-m_{i}+1, n_{i+1}, \ldots, n_{r}}^{(\alpha)}\left(x_{1}, \ldots, x_{r}\right) \\
& =\left(n_{i}-m_{i}+1\right) U_{n_{1}, \ldots, n_{i-1}, n_{i}-m_{i}+1, n_{i+1}, \ldots, n_{r}}^{(\alpha)}\left(x_{1}, \ldots, x_{r}\right),
\end{aligned}
$$

$$
\begin{aligned}
\sum_{i=1}^{r} m_{i} & x_{i} \frac{\partial}{\partial x_{i}} \\
& \times U_{n_{1}, \ldots, n_{i-1}, n_{i}-m_{i}+1, n_{i+1}, \ldots, n_{r}}^{(\alpha)}\left(x_{1}, \ldots, x_{r}\right) \\
= & \sum_{i=1}^{r}\left(n_{i}-m_{i}+1\right) \\
& \quad \times U_{n_{1}, \ldots, n_{i-1}, n_{i}-m_{i}+1, n_{i+1}, \ldots, n_{r}}^{(\alpha)}\left(x_{1}, \ldots, x_{r}\right) .
\end{aligned}
$$

for $n_{i} \geq m_{i}-1, i=1,2, \ldots, r ; n_{j} \geq 0, j \neq i$.

Remark 20. As result of Theorem 11, the multivariable polynomials $U_{n_{1}, \ldots, n_{r}}^{(\alpha)}\left(x_{1}, \ldots, x_{r}\right)$ have the following addition formula:

$$
\begin{aligned}
U_{n_{1}, \ldots, n_{r}}^{(\alpha+\beta)}\left(x_{1}, \ldots, x_{r}\right)=\sum_{k_{1}=0}^{n_{1}} \ldots \sum_{k_{r}=0}^{n_{r}} & U_{n_{1}-k_{1}, \ldots, n_{r}-k_{r}}^{(\alpha)}\left(x_{1}, \ldots, x_{r}\right) \\
& \times U_{k_{1}, \ldots, k_{r}}^{(\beta)}\left(x_{1}, \ldots, x_{r}\right) .
\end{aligned}
$$

5.2.1. The Case of $m_{i}=1, i=1,2, \ldots, r$ in Section 5.2. In this case, we get a $r$-variable analogue of Lagrange polynomials (see [14]) which is different from that in [10]

$$
\begin{aligned}
& \left(1-x_{1} t_{1}-\cdots-x_{r} t_{r}\right)^{-\alpha} \\
& \quad=\sum_{n_{1}, \ldots, n_{r}=0}^{\infty} G_{n_{1}, \ldots, n_{r}}^{(\alpha)}\left(x_{1}, \ldots, x_{r}\right) t_{1}^{n_{1}} \cdots t_{r}^{n_{r}} .
\end{aligned}
$$

They are given explicitly by

$$
G_{n_{1}, \ldots, n_{r}}^{(\alpha)}\left(x_{1}, \ldots, x_{r}\right)=\frac{(\alpha)_{n_{1}+\cdots+n_{r}}}{n_{1} ! \cdots n_{r} !} x_{1}^{n_{1}} \cdots x_{r}^{n_{r}} .
$$

Remark 21. From Corollaries 8 and 9, the multivariable polynomials $G_{n_{1}, \ldots, n_{r}}^{(\alpha)}\left(x_{1}, \ldots, x_{r}\right)$ satisfy

$$
n_{i} G_{n_{1}, \ldots, n_{r}}^{(\alpha)}\left(x_{1}, \ldots, x_{r}\right)=x_{i} \frac{\partial}{\partial x_{i}} G_{n_{1}, \ldots, n_{r}}^{(\alpha)}\left(x_{1}, \ldots, x_{r}\right)
$$

for $i=1,2, \ldots, r$ and

$$
\begin{aligned}
\sum_{i=1}^{r} n_{i} G_{n_{1}, \ldots, n_{r}}^{(\alpha)}\left(x_{1}, \ldots, x_{r}\right) & \\
& =\sum_{i=1}^{r} x_{i} \frac{\partial}{\partial x_{i}} G_{n_{1}, \ldots, n_{r}}^{(\alpha)}\left(x_{1}, \ldots, x_{r}\right) .
\end{aligned}
$$

Remark 22. By Theorem 11, the multivariable polynomials $G_{n_{1}, \ldots, n_{r}}^{(\alpha)}\left(x_{1}, \ldots, x_{r}\right)$ have the following addition formula:

$$
\begin{aligned}
G_{n_{1}, \ldots, n_{r}}^{(\alpha+\beta)}\left(x_{1}, \ldots, x_{r}\right)=\sum_{k_{1}=0}^{n_{1}} \ldots \sum_{k_{r}=0}^{n_{r}} & G_{n_{1}-k_{1}, \ldots, n_{r}-k_{r}}^{(\alpha)}\left(x_{1}, \ldots, x_{r}\right) \\
& \times G_{k_{1}, \ldots, k_{r}}^{(\beta)}\left(x_{1}, \ldots, x_{r}\right) .
\end{aligned}
$$


Remark 23. From Theorem 12, expansions of $G_{n_{1}, \ldots, n_{r}}^{(\alpha)}\left(x_{1}\right.$, $\left.\ldots, x_{r}\right)$ in series of Legendre, Gegenbauer, Hermite, and Laguerre polynomials are given by

$$
\begin{aligned}
& G_{n_{1}, \ldots, n_{r}}^{(\alpha)}\left(x_{1}, \ldots, x_{r}\right) \\
& =\prod_{i=1}^{r}\left\{\sum_{s_{i}=0}^{\left[n_{i} / 2\right]} \frac{\left(2 n_{i}-4 s_{i}+1\right)}{s_{i} !(3 / 2)_{n_{i}-s_{i}}} P_{n_{i}-2 s_{i}}\left(\frac{x_{i}}{2}\right)\right\} \\
& \times(\alpha)_{n_{1}+\cdots+n_{r}}, \\
& G_{n_{1}, \ldots, n_{r}}^{(\alpha)}\left(x_{1}, \ldots, x_{r}\right) \\
& =\prod_{i=1}^{r}\left\{\sum_{s_{i}=0}^{\left[n_{i} / 2\right]} \frac{\left(v_{i}+n_{i}-2 s_{i}\right)}{s_{i} !\left(v_{i}\right)_{n_{i}-s_{i}+1}} C_{n_{i}-2 s_{i}}^{v_{i}}\left(\frac{x_{i}}{2}\right)\right\} \\
& \times(\alpha)_{n_{1}+\cdots+n_{r}}, \\
& G_{n_{1}, \ldots, n_{r}}^{(\alpha)}\left(x_{1}, \ldots, x_{r}\right) \\
& =\prod_{i=1}^{r}\left\{\sum_{s_{i}=0}^{\left[n_{i} / 2\right]} \frac{H_{n_{i}-2 s_{i}}\left(x_{i} / 2\right)}{s_{i} !\left(n_{i}-2 s_{i}\right) !}\right\}(\alpha)_{n_{1}+\cdots+n_{r}}, \\
& G_{n_{1}, \ldots, n_{r}}^{(\alpha)}\left(x_{1}, \ldots, x_{r}\right) \\
& =\prod_{i=1}^{r}\left\{\sum_{s_{i}=0}^{n_{i}} \frac{\left(\beta_{i}+1\right)_{n_{i}} 2^{n_{i}}(-1)^{s_{i}}}{\left(n_{i}-s_{i}\right) !\left(\beta_{i}+1\right)_{s_{i}}} L_{s_{i}}^{\left(\beta_{i}\right)}\left(\frac{x_{i}}{2}\right)\right\} \\
& \times(\alpha)_{n_{1}+\cdots+n_{r}} .
\end{aligned}
$$

We can discuss some generating functions for the multivariable polynomials $G_{n_{1}, \ldots, n_{r}}^{(\alpha)}\left(x_{1}, \ldots, x_{r}\right)$.

Theorem 24. For the polynomials $G_{n_{1}, \ldots, n_{r}}^{(\alpha)}\left(x_{1}, \ldots, x_{r}\right)$, the following generating function holds true for $k \in \mathbb{N}_{0}$ :

$$
\begin{aligned}
\sum_{n_{1}, \ldots, n_{r}=0}^{\infty} G_{n_{1}, \ldots, n_{r}}^{(\alpha)}\left(x_{1}, \ldots, x_{r}\right)\left[k+n_{i}\right]_{m} z_{1}^{n_{1}} \cdots z_{r}^{n_{r}} \\
=m !\left(1-x_{1} z_{1}-\cdots-x_{r} z_{r}\right)^{-\alpha} \\
\quad \times g_{m}^{(\alpha,-k)}\left(\frac{x_{i} z_{i}}{1-x_{1} z_{1}-\cdots-x_{r} z_{r}},-1\right),
\end{aligned}
$$

for each $i=1,2, \ldots, r$, where

$$
[k]_{m}:=k(k-1) \cdots(k-m+1), \quad m \in \mathbb{N},[k]_{0}=1
$$

and $\operatorname{g}_{n}^{(\alpha, \beta)}(x, y)$ denotes the classical Lagrange polynomials defined by [14]

$$
\begin{gathered}
(1-x t)^{-\alpha}(1-y t)^{-\beta}=\sum_{n=0}^{\infty} \mathrm{g}_{n}^{(\alpha, \beta)}(x, y) t^{n} \\
\left(\alpha, \beta \in \mathbb{C} ;|t|<\min \left\{|x|^{-1},|y|^{-1}\right\}\right) .
\end{gathered}
$$

Proof. Fix $i=1,2, \ldots, r$. Let $\mathscr{C}_{\xi_{i}}$ and $\mathscr{C}_{\xi_{i}}^{*}$ denote the circles of radius $\varepsilon_{i}$, centered $\xi_{i}=z_{i}$ and $\xi_{i}=0$, respectively, where

$$
0<\varepsilon_{i}<\left|x_{i}\right|^{-1}\left|1-\sum_{k=1}^{r} x_{k} z_{k}\right| .
$$

Here these circles are described in the positive direction. By Cauchy's Integral Formula, we have

$$
\begin{aligned}
D_{z_{i}}^{m}\{ & \left.\left(1-x_{1} z_{1}-\cdots-x_{r} z_{r}\right)^{-\alpha} z_{i}^{k}\right\} \\
= & \frac{m !}{2 \pi i} \oint_{\mathscr{C}_{\xi_{i}}} \frac{\xi_{i}^{k}\left(1-x_{1} z_{1}-\cdots-x_{i} \xi_{i}-\cdots-x_{r} z_{r}\right)^{-\alpha}}{\left(\xi_{i}-z_{i}\right)^{m+1}} d \xi_{i} \\
= & \frac{m !}{2 \pi i} \\
& \times \oint_{\mathscr{C}_{\xi_{i}}^{*}} \frac{\left(\xi_{i}+z_{i}\right)^{k}\left(1-x_{1} z_{1}-\cdots-x_{i}\left(\xi_{i}+z_{i}\right)-\cdots-x_{r} z_{r}\right)^{-\alpha}}{\xi_{i}^{m+1}} d \xi_{i} \\
= & \frac{m !}{2 \pi i} z_{i}^{k}\left(1-x_{1} z_{1}-\cdots-x_{r} z_{r}\right)^{-\alpha} \\
& \times \oint_{\mathscr{C}_{\xi_{i}}^{*}} \frac{\left(1+\xi_{i} / z_{i}\right)^{k}}{\xi_{i}^{m+1}}\left(1-\frac{x_{i} \xi_{i}}{1-x_{1} z_{1}-\cdots-x_{r} z_{r}}\right)^{-\alpha} d \xi_{i} \\
= & m ! z_{i}^{k}\left(1-x_{1} z_{1}-\cdots-x_{r} z_{r}\right)^{-\alpha} \\
& \times \mathrm{g}_{m}^{(\alpha,-k)}\left(\frac{x_{i}}{1-x_{1} z_{1}-\cdots-x_{r} z_{r}},-\frac{1}{z_{i}}\right) \\
= & m ! z_{i}^{k-m}\left(1-x_{1} z_{1}-\cdots-x_{r} z_{r}\right)^{-\alpha} \\
& \times \mathrm{g}_{m}^{(\alpha,-k)}\left(\frac{x_{i} z_{i}}{1-x_{1} z_{1}-\cdots-x_{r} z_{r}},-1\right) .
\end{aligned}
$$

On the other hand, we can write that

$$
\begin{aligned}
D_{z_{i}}^{m} & \left\{\left(1-x_{1} z_{1}-\cdots-x_{r} z_{r}\right)^{-\alpha} z_{i}^{k}\right\} \\
& =D_{z_{i}}^{m}\left\{z_{i}^{k} \sum_{n_{1}, \ldots, n_{r}=0}^{\infty} G_{n_{1}, \ldots, n_{r}}^{(\alpha)}\left(x_{1}, \ldots, x_{r}\right) z_{1}^{n_{1}} \cdots z_{r}^{n_{r}}\right\} \\
& =\sum_{n_{1}, \ldots, n_{r}=0}^{\infty} G_{n_{1}, \ldots, n_{r}}^{(\alpha)}\left(x_{1}, \ldots, x_{r}\right) \frac{d^{m}}{d z_{i}^{m}} z_{1}^{n_{1}} \cdots z_{i}^{n_{i}+k} \cdots z_{r}^{n_{r}} \\
& =z_{i}^{k-m} \sum_{n_{1}, \ldots, n_{r}=0}^{\infty} G_{n_{1}, \ldots, n_{r}}^{(\alpha)}\left(x_{1}, \ldots, x_{r}\right)\left[k+n_{i}\right]_{m} z_{1}^{n_{1}} \cdots z_{r}^{n_{r}} .
\end{aligned}
$$

From (74) and (75), we see that

$$
\begin{aligned}
\sum_{n_{1}, \ldots, n_{r}=0}^{\infty} G_{n_{1}, \ldots, n_{r}}^{(\alpha)}\left(x_{1}, \ldots, x_{r}\right)\left[k+n_{i}\right]_{m} z_{1}^{n_{1}} \cdots z_{r}^{n_{r}} \\
=m !\left(1-x_{1} z_{1}-\cdots-x_{r} z_{r}\right)^{-\alpha} \\
\quad \times \mathrm{g}_{m}^{(\alpha,-k)}\left(\frac{x_{i} z_{i}}{1-x_{1} z_{1}-\cdots-x_{r} z_{r}},-1\right) .
\end{aligned}
$$

Corollary 25. From (71), one observes that

$$
\begin{aligned}
{\left[n_{i}+m\right]_{m} } & =\left(n_{i}+m\right)\left(n_{i}+m-1\right) \cdots\left(n_{i}+1\right) \\
& =\left(n_{i}+1\right)_{m}
\end{aligned}
$$


for each $i=1,2, \ldots, r$. By setting $k=m$ in (70) and then using (77), one has

$$
\begin{aligned}
\sum_{n_{1}, \ldots, n_{r}=0}^{\infty}\left(n_{i}+1\right)_{m} G_{n_{1}, \ldots, n_{r}}^{(\alpha)}\left(x_{1}, \ldots, x_{r}\right) z_{1}^{n_{1}} \cdots z_{r}^{n_{r}} \\
=m !\left(1-x_{1} z_{1}-\cdots-x_{r} z_{r}\right)^{-\alpha} \\
\quad \times g_{m}^{(\alpha,-m)}\left(\frac{x_{i} z_{i}}{1-x_{1} z_{1}-\cdots-x_{r} z_{r}},-1\right) .
\end{aligned}
$$

5.2.2. The Case of $m_{i}=i, i=1,2, \ldots, r$ in Section 5.2. This case reduces to a multivariable analogue of Lagrange-Hermite polynomials

$$
\begin{aligned}
& \left(1-x_{1} t_{1}-x_{2} t_{2}^{2}-\cdots-x_{r} t_{r}^{r}\right)^{-\alpha} \\
& \quad=\sum_{n_{1}, \ldots, n_{r}=0}^{\infty} H_{n_{1}, \ldots, n_{r}}^{(\alpha)}\left(x_{1}, \ldots, x_{r}\right) t_{1}^{n_{1}} \cdots t_{r}^{n_{r}},
\end{aligned}
$$

which is different from that in [6].

Remark 26. By Corollaries 8 and 9, the multivariable polynomials $H_{n_{1}, \ldots, n_{r}}^{(\alpha)}\left(x_{1}, \ldots, x_{r}\right)$ satisfy

$$
\begin{aligned}
& i x_{i} \frac{\partial}{\partial x_{i}} H_{n_{1}, \ldots, n_{i-1}, n_{i}-i+1, n_{i+1}, \ldots, n_{r}}^{(\alpha)}\left(x_{1}, \ldots, x_{r}\right) \\
&=\left(n_{i}-i+1\right) H_{n_{1}, \ldots, n_{i-1}, n_{i}-i+1, n_{i+1}, \ldots, n_{r}}^{(\alpha)}\left(x_{1}, \ldots, x_{r}\right) \\
& \sum_{i=1}^{r} i x_{i} \frac{\partial}{\partial x_{i}} H_{n_{1}, \ldots, n_{i-1}, n_{i}-i+1, n_{i+1}, \ldots, n_{r}}^{(\alpha)}\left(x_{1}, \ldots, x_{r}\right) \\
&=\sum_{i=1}^{r}\left(n_{i}-i+1\right) \\
& \quad \times H_{n_{1}, \ldots, n_{i-1}, n_{i}-i+1, n_{i+1}, \ldots, n_{r}}^{(\alpha)}\left(x_{1}, \ldots, x_{r}\right) .
\end{aligned}
$$

for $n_{i} \geq i-1, \quad i=1,2, \ldots, r ; n_{j} \geq 0, j \neq i$.

Remark 27. From Theorem 11, the multivariable polynomials $H_{n_{1}, \ldots, n_{r}}^{(\alpha)}\left(x_{1}, \ldots, x_{r}\right)$ have the following addition formula:

$$
\begin{aligned}
& H_{n_{1}, \ldots, n_{r}}^{(\alpha+\beta)}\left(x_{1}, \ldots, x_{r}\right) \\
& =\sum_{k_{1}=0}^{n_{1}} \ldots \sum_{k_{r}=0}^{n_{r}} H_{n_{1}-k_{1}, \ldots, n_{r}-k_{r}}^{(\alpha)}\left(x_{1}, \ldots, x_{r}\right) \\
& \times H_{k_{1}, \ldots, k_{r}}^{(\beta)}\left(x_{1}, \ldots, x_{r}\right) .
\end{aligned}
$$

Furthermore, setting $m_{i}=i, x_{i}=0, y_{i}=-x_{i}$, $i=1,2, \ldots, r$ in Corollary 5 , we obtain the following class of bilinear generating functions for the polynomials $H_{n_{1}, \ldots, n_{r}}^{(\alpha)}\left(x_{1}, \ldots, x_{r}\right)$.
Remark 28. If

$$
\begin{aligned}
& \Xi_{\lambda, \eta, \alpha, \beta, \gamma}^{\mathbf{n , p}}\left(x_{1}, \ldots, x_{r} ; u_{1}, \ldots, u_{r} ; \mathbf{w}\right) \\
& :=\sum_{k_{1}=0}^{\left[n_{1} / p_{1}\right]} \cdots \sum_{k_{r}=0}^{\left[n_{r} / p_{r}\right]} a_{k_{1}, \ldots, k_{r}} H_{n_{1}-p_{1} k_{1}, \ldots, n_{r}-p_{r} k_{r}}^{(\alpha+\beta)}\left(x_{1}, \ldots, x_{r}\right) \\
& \quad \times H_{\lambda_{1}+\eta_{1} k_{1}, \ldots, \lambda_{r}+\eta_{r} k_{r}}^{(\gamma)}\left(u_{1}, \ldots, u_{r}\right) w_{1}^{k_{1}} \cdots w_{r}^{k_{r}},
\end{aligned}
$$

where $a_{k_{1}, \ldots, k_{r}} \neq 0 ; p_{i} \in \mathbb{N} ; n_{i}, \lambda_{i}, \eta_{i} \in \mathbb{N}_{0}, i=1,2, \ldots, r$, and $\lambda=\left(\lambda_{1}, \ldots, \lambda_{r}\right), \eta=\left(\eta_{1}, \ldots, \eta_{r}\right), \mathbf{w}=\left(w_{1}, \ldots, w_{r}\right)$, then we have

$$
\begin{gathered}
\sum_{l_{1}=0}^{n_{1}} \ldots \sum_{l_{r}=0}^{n_{r}} \sum_{k_{1}=0}^{\left[l_{1} / p_{1}\right]} \ldots \sum_{k_{r}=0}^{\left[l_{r} / p_{r}\right]} a_{k_{1}, \ldots, k_{r}} H_{n_{1}-l_{1}, \ldots, n_{r}-l_{r}}^{(\alpha)}\left(x_{1}, \ldots, x_{r}\right) \\
\times H_{l_{1}-p_{1} k_{1}, \ldots, l_{r}-p_{r} k_{r}}^{(\beta)}\left(x_{1}, \ldots, x_{r}\right) \\
\times H_{\lambda_{1}+\eta_{1} k_{1}, \ldots, \lambda_{r}+\eta_{r} k_{r}}^{(\gamma)}\left(u_{1}, \ldots, u_{r}\right) \\
\times w_{1}^{k_{1}} \ldots w_{r}^{k_{r}} \\
=\Xi_{\lambda, \eta, \alpha, \beta, \gamma}^{\mathbf{n}, \mathbf{p}}\left(x_{1}, \ldots, x_{r} ; u_{1}, \ldots, u_{r} ; \mathbf{w}\right) .
\end{gathered}
$$

\section{References}

[1] H. W. Gould, "Inverse series relation and other expansions involving Humbert polynomials," Duke Mathematical Journal, vol. 32, pp. 697-711, 1965.

[2] H. M. Srivastava and H. L. A. Manocha, Treatise on Generating Functions, Halsted Press, Ellis Horwood Limited, John Wiley and Sons, Chichester, UK, 1984.

[3] J. P. Singhal, "A note on generalized Humbert polynomials," Glasnik Matematicki III, vol. 5, no. 25, pp. 241-245, 1970.

[4] D. Zeitlin, "On a class of polynomials obtained from generalized Humbert polynomials," Proceedings of the American Mathematical Society, vol. 18, pp. 28-34, 1967.

[5] R. Aktaş, R. Şahin, and A. Altın, "On a multivariable extension of Humbert polynomials," Applied Mathematics and Computation, vol. 218, pp. 662-666, 2011.

[6] A. Altın and E. Erkuş, "On a multivariable extension of the Lagrange-Hermite polynomials," Integral Transforms and Special Functions, vol. 17, no. 4, pp. 239-244, 2006.

[7] A. Altın, E. Erkuş, and M. A. Özarslan, "Families of linear generating functions for polyno- mials in two variables," Integral Transforms and Special Functions, vol. 17, no. 5, pp. 315-320, 2006.

[8] E. Erkuş and H. M. Srivastava, "A unfied presentation of some families of multivariable polynomials," Integral Transforms and Special Functions, vol. 17, pp. 267-273, 2006.

[9] E. Özergin, M. A. Özarslan, and H. M. Srivastava, "Some families of generating functions for a class of bivariate polynomials," Mathematical and Computer Modelling, vol. 50, pp. 1113-1120, 2009.

[10] W.-C. C. Chan, C.-J. Chyan, and H. M. Srivastava, "The Lagrange polynomials in several variables," Integral Transforms and Special Functions, vol. 12, no. 2, pp. 139-148, 2001. 
[11] E. D. Rainville, Special Functions, The Macmillan Company, New York, NY, USA, 1960.

[12] M. A. Khan and G. S. Kahmmash, "A study of a two variables Gegenbauer polynomials," Applied Mathematical Sciences, vol. 2, no. 13, pp. 639-657, 2008.

[13] G. Lauricella, "Sulle funzioni ipergeometriche a più variabili," Rendiconti del Circolo Matematico di Palermo, vol. 7, pp. 111-158, 1893.

[14] A. Erdélyi, W. Magnus, F. Oberhettinger, and F. G. Tricomi, Higher Transcendental Functions, vol. 3, McGraw-Hill Book Company, New York, NY, USA, 1955. 


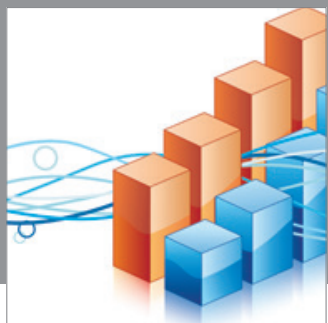

Advances in

Operations Research

mansans

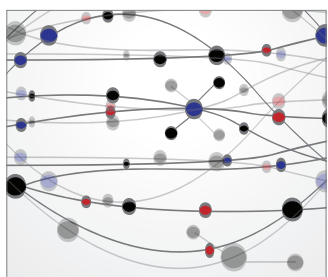

The Scientific World Journal
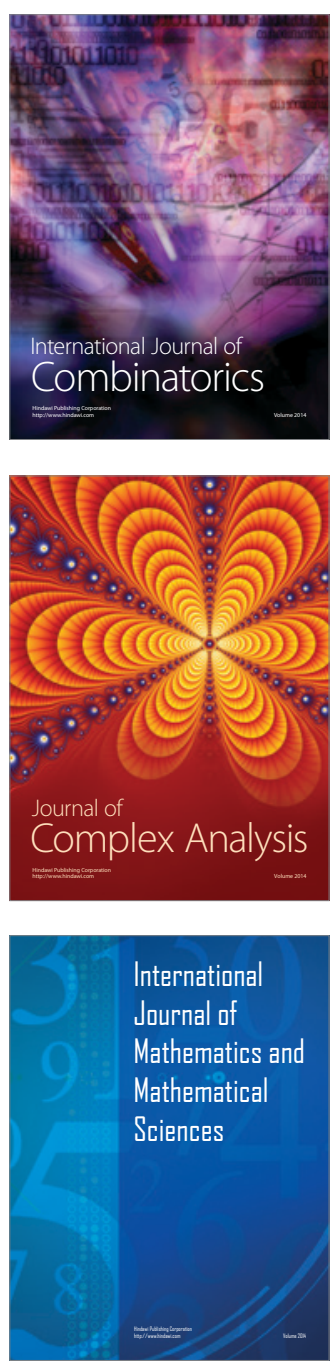
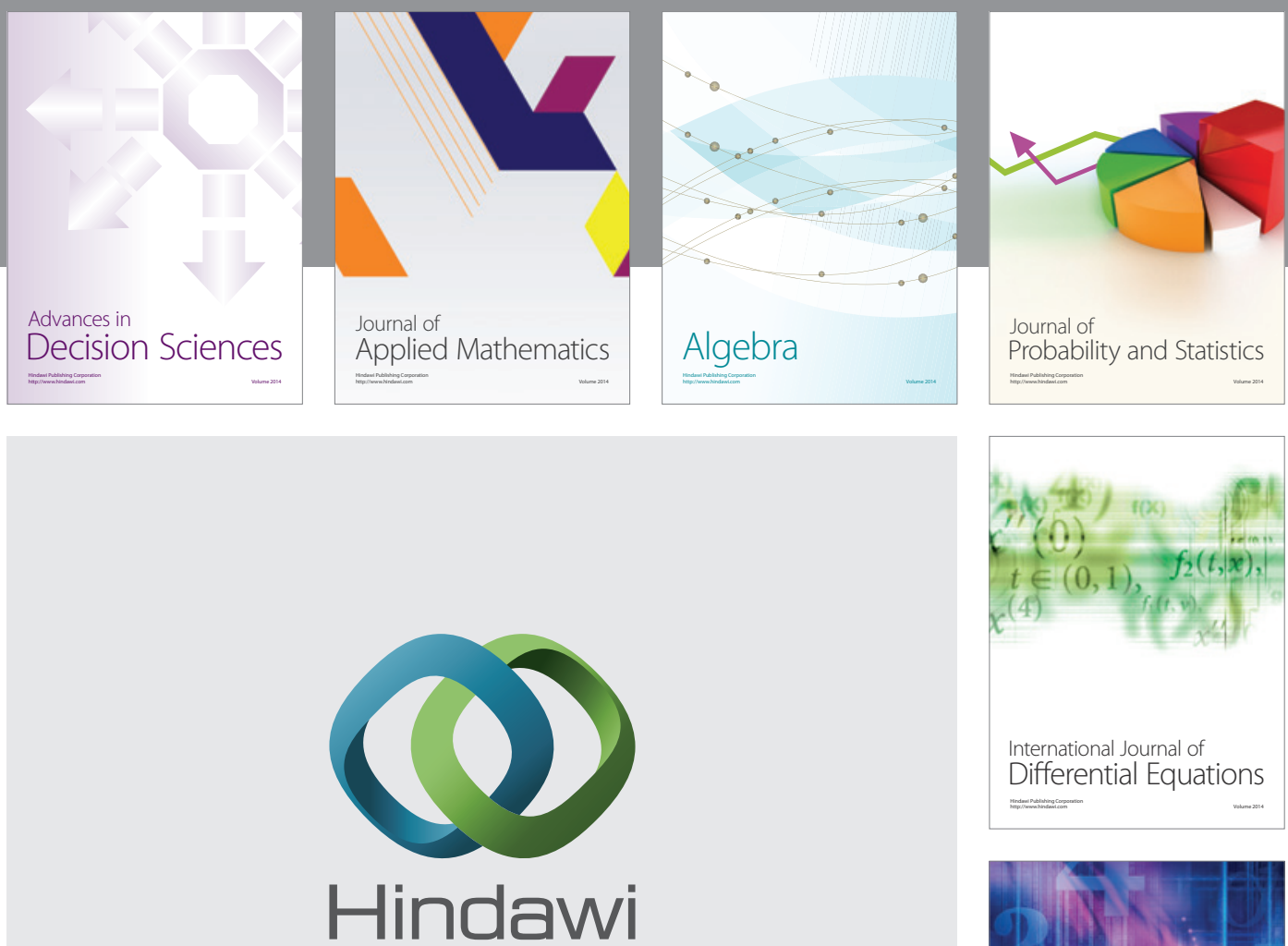

Submit your manuscripts at http://www.hindawi.com
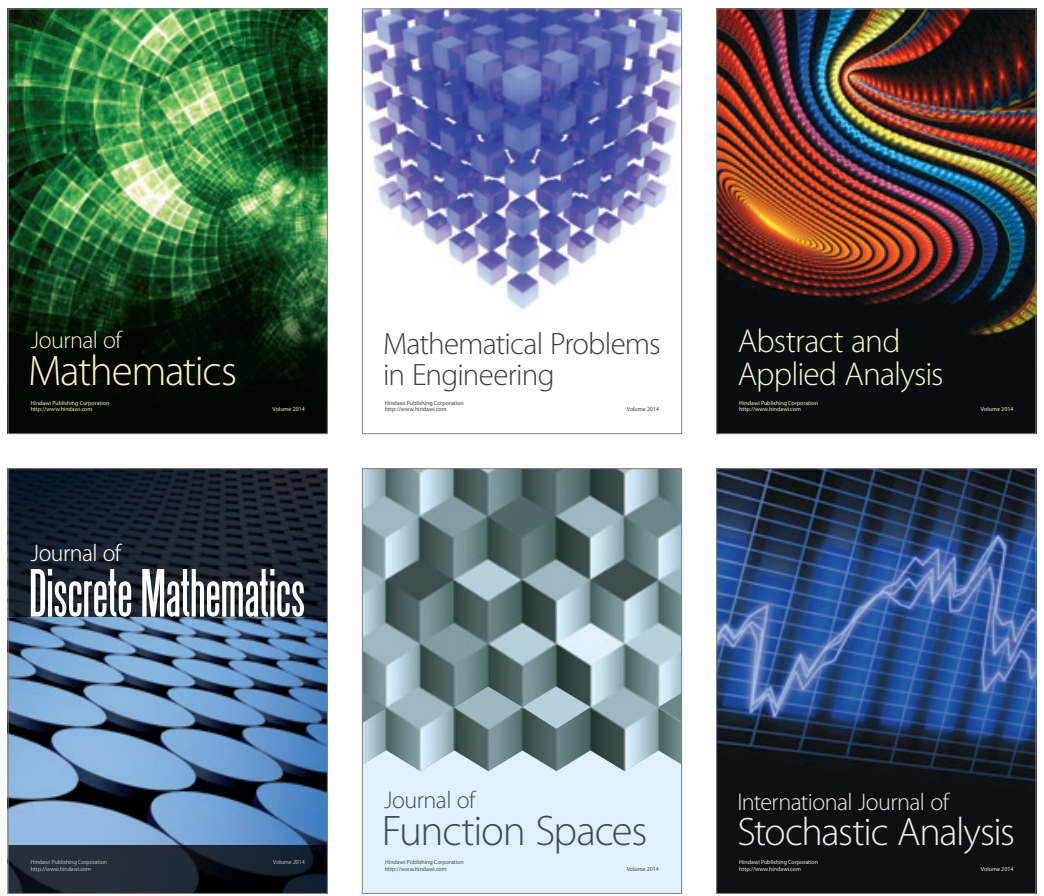

Journal of

Function Spaces

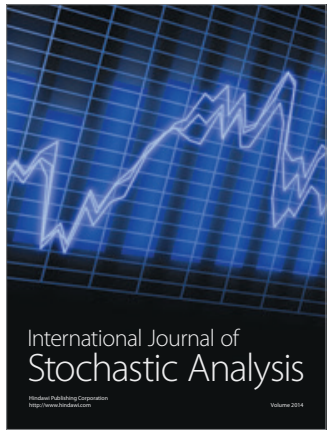

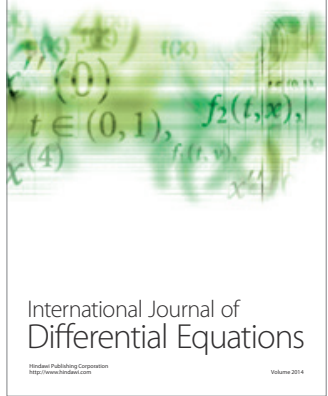
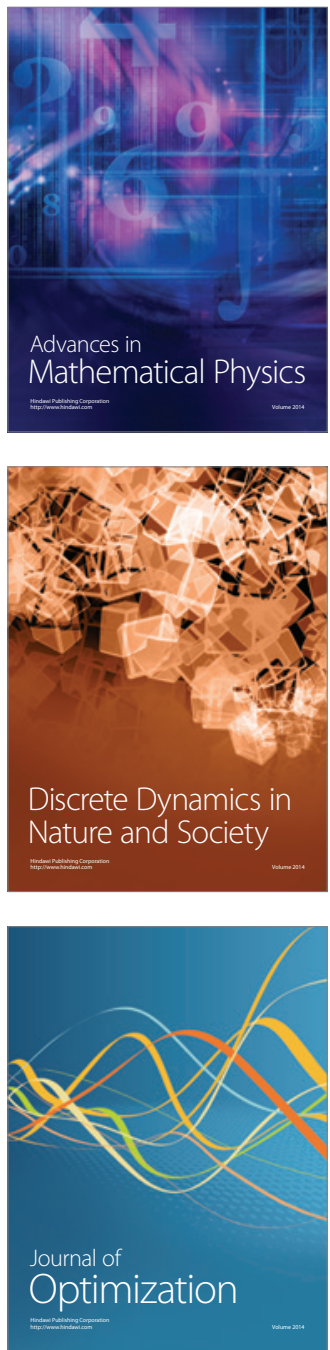